\title{
Dark matter, electroweak phase transition, and gravitational waves in the type II two-Higgs-doublet model with a singlet scalar field
}

\author{
Xiao-Fang Han, ${ }^{1}$ Lei Wang $\odot,{ }^{1, *}$ and Yang Zhang ${ }^{2,3}$ \\ ${ }^{1}$ Department of Physics, Yantai University, Yantai 264005, People's Republic of China \\ ${ }^{2}$ School of Physics and Microelectronics, Zhengzhou University, ZhengZhou 450001, People's Republic of China \\ ${ }^{3}$ ARC Centre of Excellence for Particle Physics at the Tera-scale, School of Physics and Astronomy, \\ Monash University, Melbourne, Victoria 3800, Australia
}

(Received 24 November 2020; accepted 20 January 2021; published 15 February 2021)

\begin{abstract}
In the framework of the type II two-Higgs-doublet model with a singlet scalar dark matter $S$, we study the dark matter observables, the electroweak phase transition, and the gravitational wave signals by such strongly first order phase transition after imposing the constraints of the LHC Higgs data. We take the heavy $C P$-even Higgs boson $H$ as the only portal between the dark matter and standard model (SM) sectors, and find that the LHC Higgs data and dark matter observables require $m_{S}$ and $m_{H}$ to be larger than $130 \mathrm{GeV}$ and $360 \mathrm{GeV}$ for $m_{A}=600 \mathrm{GeV}$ in the case of the $125 \mathrm{GeV}$ Higgs boson with the SM-like coupling. Next, we carve out some parameter space where a strongly first order electroweak phase transition can be achieved, and find benchmark points for which the amplitudes of gravitational wave spectra reach the sensitivities of the future gravitational wave detectors.
\end{abstract}

DOI: $10.1103 /$ PhysRevD.103.035012

\section{INTRODUCTION}

The weakly interacting massive particle is a primary candidate for dark matter (DM) in the present Universe. Many extensions of the SM have been proposed to provide a candidate for DM, and one simple extension is to add a singlet scalar DM to the type II two-Higgs-doublet model (2HDM) [1-3]. The type II 2HDM contains two $C P$-even states, $h$ and $H$, one neutral pseudoscalar $A$, two charged scalars $H^{ \pm}$, and one $C P$-even singlet scalar $S$ as the candidate for DM [4-14].

In the type II 2HDM, the Yukawa couplings of the downtype quark and lepton can both be enhanced by a factor of $\tan \beta$. Therefore, the flavor observables and the LHC searches for the Higgs boson can impose strong constraints on the type II $2 \mathrm{HDM}$. In the type II $2 \mathrm{HDM}$ with a singlet scalar DM (2HDMIID), the two $C P$-even states $h$ and $H$ may be portals between the DM and SM sectors, and there is plentiful parameter space satisfying the direct and indirect experimental constraints of DM. The scalar potential of 2HDMIID contains the original potential of type II 2HDM and one including DM. For the appropriate Higgs boson mass spectrum and coupling constants, the type II 2HDM can trigger a strong first order electroweak phase transition (SFOEWPT) in the early Universe [15-19], which

\footnotetext{
* Corresponding author. leiwang@ytu.edu.cn

Published by the American Physical Society under the terms of the Creative Commons Attribution 4.0 International license. Further distribution of this work must maintain attribution to the author(s) and the published article's title, journal citation, and DOI. Funded by SCOAP .
}

is required by a successful explanation of the observed baryon asymmetry of the Universe (BAU) [20] and can produce primordial gravitational wave $(\mathrm{GW})$ signals [21].

In this paper, we first examine the parameter space of the 2HDMIID using the recent LHC Higgs data and DM observables. After imposing various theoretical and experimental constraints, we analyze whether a SFOEWPT is achievable in the 2HDMIID, and discuss the resultant GW signals and their detectability at the future GW detectors, such as LISA [22], Taiji [23], TianQin [24], Big Bang Observer (BBO) [25], DECi-hertz Interferometer GW Observatory (DECIGO) [25], and Ultimate-DECIGO [26].

Our work is organized as follows: In Sec. II we give a brief introduction to the 2HDMIID. In Secs. III and IV, we show the allowed parameter space after imposing the limits of the LHC Higgs data and DM observables. In Sec. V, we examine the parameter space leading to a SFOEWPT and the corresponding GW signal. Finally, we give our conclusions in Sec. VI.

\section{TYPE II TWO-HIGGS-DOUBLET MODEL WITH A SCALAR DARK MATTER}

The scalar potential of 2HDMIID is given as [27]

$$
\begin{aligned}
\mathcal{V}_{\text {tree }}= & m_{11}^{2}\left(\Phi_{1}^{\dagger} \Phi_{1}\right)+m_{22}^{2}\left(\Phi_{2}^{\dagger} \Phi_{2}\right)-\left[m_{12}^{2}\left(\Phi_{1}^{\dagger} \Phi_{2}+\text { H.c. }\right)\right] \\
& +\frac{\lambda_{1}}{2}\left(\Phi_{1}^{\dagger} \Phi_{1}\right)^{2}+\frac{\lambda_{2}}{2}\left(\Phi_{2}^{\dagger} \Phi_{2}\right)^{2}+\lambda_{3}\left(\Phi_{1}^{\dagger} \Phi_{1}\right)\left(\Phi_{2}^{\dagger} \Phi_{2}\right) \\
& +\lambda_{4}\left(\Phi_{1}^{\dagger} \Phi_{2}\right)\left(\Phi_{2}^{\dagger} \Phi_{1}\right)+\left[\frac{\lambda_{5}}{2}\left(\Phi_{1}^{\dagger} \Phi_{2}\right)^{2}+\text { H.c. }\right] \\
& +\frac{1}{2} S^{2}\left(\kappa_{1} \Phi_{1}^{\dagger} \Phi_{1}+\kappa_{2} \Phi_{2}^{\dagger} \Phi_{2}\right)+\frac{m_{0}^{2}}{2} S^{2}+\frac{\lambda_{S}}{4 !} S^{4} .
\end{aligned}
$$


Here we discuss the $C P$-conserving model in which all $\lambda_{i}$, $\kappa_{i}$, and $m_{12}^{2}$ are real. The $S$ is a real singlet scalar field, and $\Phi_{1}$ and $\Phi_{2}$ are complex Higgs doublets with hypercharge $Y=1$ :

$$
\begin{aligned}
& \Phi_{1}=\left(\begin{array}{c}
\phi_{1}^{+} \\
\frac{1}{\sqrt{2}}\left(v_{1}+\phi_{1}^{0}+i a_{1}\right)
\end{array}\right), \\
& \Phi_{2}=\left(\begin{array}{c}
\phi_{2}^{+} \\
\frac{1}{\sqrt{2}}\left(v_{2}+\phi_{2}^{0}+i a_{2}\right)
\end{array}\right) .
\end{aligned}
$$

$v_{1}$ and $v_{2}$ are the electroweak vacuum expectation values (VEVs) with $v^{2}=v_{1}^{2}+v_{2}^{2}=(246 \mathrm{GeV})^{2}$, and the ratio of the two VEVs is defined as $\tan \beta=v_{2} / v_{1}$. The linear and cubic terms of the $S$ field are forbidden by a $Z_{2}^{\prime}$ symmetry, under which $S \rightarrow-S$. The $S$ is a possible DM candidate since it does not acquire a VEV. After spontaneous electroweak symmetry breaking, the remaining physical states are three neutral $C P$-even states $h, H$, and $S$, one neutral pseudoscalar $A$, and two charged scalars $H^{ \pm}$.

We can obtain the DM mass and the cubic interactions with the neutral Higgs bosons from Eq. (1),

$$
\begin{aligned}
m_{S}^{2} & =m_{0}^{2}+\frac{1}{2} \kappa_{1} v^{2} \cos ^{2} \beta+\frac{1}{2} \kappa_{2} v^{2} \sin ^{2} \beta, \\
-\lambda_{h} v S^{2} h / 2 & \equiv-\left(-\kappa_{1} \sin \alpha \cos \beta+\kappa_{2} \cos \alpha \sin \beta\right) v S^{2} h / 2, \\
-\lambda_{H} v S^{2} H / 2 & \equiv-\left(\kappa_{1} \cos \alpha \cos \beta+\kappa_{2} \sin \alpha \sin \beta\right) v S^{2} H / 2,
\end{aligned}
$$

with $\alpha$ being the mixing angle of $h$ and $H$.

The Yukawa interactions are written as

$-\mathcal{L}=Y_{u 2} \bar{Q}_{L} \tilde{\Phi}_{2} u_{R}+Y_{d 1} \bar{Q}_{L} \Phi_{1} d_{R}+Y_{\ell 1} \bar{L}_{L} \Phi_{1} e_{R}+$ H.c.

where $Q_{L}^{T}=\left(u_{L}, d_{L}\right), L_{L}^{T}=\left(\nu_{L}, l_{L}\right), \tilde{\Phi}_{1,2}=i \tau_{2} \Phi_{1,2}^{*}$, and $Y_{u 2}, Y_{d 1}$, and $Y_{\ell 1}$ are $3 \times 3$ matrices in family space.

The Yukawa couplings of the neutral Higgs bosons normalized to the SM are given by

$$
\begin{aligned}
y_{h}^{f_{i}}= & {\left[\sin (\beta-\alpha)+\cos (\beta-\alpha) \kappa_{f}\right], } \\
y_{H}^{f_{i}}= & {\left[\cos (\beta-\alpha)-\sin (\beta-\alpha) \kappa_{f}\right], } \\
y_{A}^{f_{i}}= & -i \kappa_{f}(\text { for } \mathrm{u}), \quad y_{A}^{f_{i}}=i \kappa_{f}(\text { for } \mathrm{d}, \ell), \\
& \text { with } \quad \kappa_{d}=\kappa_{\ell} \equiv-\tan \beta, \quad \kappa_{u} \equiv 1 / \tan \beta .
\end{aligned}
$$

The charged Higgs boson has the following Yukawa interactions,

$$
\begin{aligned}
\mathcal{L}_{Y}= & -\frac{\sqrt{2}}{v} H^{+}\left\{\overline { u } _ { i } \left[\kappa_{d}\left(V_{\mathrm{CKM}}\right)_{i j} m_{d j} P_{R}\right.\right. \\
& \left.\left.-\kappa_{u} m_{u i}\left(V_{\mathrm{CKM}}\right)_{i j} P_{L}\right] d_{j}+\kappa_{\ell} \bar{\nu} m_{\ell} P_{R} \ell\right\}+ \text { H.c. }
\end{aligned}
$$

where $i, j=1,2,3$.

The neutral Higgs boson couplings with the gauge bosons normalized to the SM are given by

$$
y_{h}^{V}=\sin (\beta-\alpha), \quad y_{H}^{V}=\cos (\beta-\alpha),
$$

where $V$ denotes $Z$ or $W$. In the type II $2 \mathrm{HDM}$, the $125 \mathrm{GeV}$ Higgs boson is allowed to have the SM-like coupling and wrong sign Yukawa coupling, where

$$
\begin{aligned}
& \qquad y_{h}^{f_{i}} \times y_{h}^{V}>0 \text { for SM-like coupling, } \\
& y_{h}^{f_{i}} \times y_{h}^{V}<0 \text { for wrong sign Yukawa coupling. } \\
& \text { III. THE EXPERIMENTAL CONSTRAINTS } \\
& \text { OF THE HIGGS DATA AT THE LHC }
\end{aligned}
$$

\section{THE EXPERIMENTAL CONSTRAINTS OF THE HIGGS DATA AT THE LHC}

\section{A. Numerical calculations}

We take the light $C P$-even Higgs boson $h$ as the SM-like Higgs boson, $m_{h}=125 \mathrm{GeV}$. The measurement of the branching fraction of $b \rightarrow s \gamma$ gives the stringent constraints on the charged Higgs mass of the type II $2 \mathrm{HDM}, m_{H^{ \pm}}>$ $570 \mathrm{GeV}$ [28]. If the $125 \mathrm{GeV}$ Higgs boson is the portal between the DM and SM sectors, it is favored to have wrong sign Yukawa coupling which can realize the isospinviolating DM interactions with nucleons and relax the bounds of direct detection of DM. However, Ref. [17] shows the wrong sign Yukawa coupling region of type II $2 \mathrm{HDM}$ is strongly restricted by the requirement of SFOEWPT. Therefore, in this paper we take the heavy $\mathrm{CP}$-even Higgs boson $H$ as the only portal between the DM and SM sectors, and focus on the case of the $125 \mathrm{GeV}$ with the SM-like coupling. The $S, T$, and $U$ oblique parameters give the stringent constraints on the mass spectrum of Higgs bosons of type II 2HDM [29-31]. One of $m_{A}$ and $m_{H}$ is around $600 \mathrm{GeV}$, and another is allowed to have a wide mass range, including low mass [30,31]. Therefore, we fix $m_{A}=600 \mathrm{GeV}$ to cause the portal $H$ to have a wide mass range.

In our calculations, we consider the following observables and constraints:

(1) The theoretical constraints. The scalar potential of the model contains one of the type II 2HDM and one of the DM sector. The vacuum stability, perturbativity, and tree-level unitarity impose constraints on the relevant parameters, which are discussed in detail in Refs. $[8,9]$. Here we employ the formulas in $[8,9]$ to implement the theoretical constraints. Compared to Refs. [8,9], there are additional factors of $\frac{1}{2}$ in the $\kappa_{1}$ term and $\kappa_{2}$ terms in this paper. In addition, we require that the potential has a global minimum at 
the point of $\left(\left\langle h_{1}\right\rangle=v_{1},\left\langle h_{2}\right\rangle=v_{2},\left\langle S_{1}\right\rangle=0\right)$. We do not consider the stability of the scalar potential at a high energy scale using the renormalization group evolution. In the SM there is only one quartic Higgs coupling, while the potential of this model has eight quartic Higgs couplings. In addition, the relations between Yukawa couplings and quark masses are different from the SM, which can be controlled by the two parameters $\beta$ and $\alpha$. Therefore, by tunning the parameters, the model should have some different properties from the SM Higgs potential. The detailed study is complicated and beyond the scope of this paper.

(2) The oblique parameters. The $S, T$, and $U$ parameters can impose stringent constraints on the mass spectrum of the Higgs bosons of 2HDM. We use 2HDMC [32] to calculate the $S, T$, and $U$ parameters. Taking the recent fit results of Ref. [33], we use the following values of $S, T$, and $U$ :

$$
\begin{aligned}
S & =0.02 \pm 0.10, \quad T=0.07 \pm 0.12, \\
U & =0.00 \pm 0.09 .
\end{aligned}
$$

The correlation coefficients are

$\rho_{S T}=0.89, \quad \rho_{S U}=-0.54, \quad \rho_{T U}=-0.83$.

(3) The flavor observables and $R_{b}$. We employ SuperIso3.4 [34] to calculate $\operatorname{Br}\left(B \rightarrow X_{s} \gamma\right)$, and $\Delta m_{B_{s}}$ is calculated following the formulas in [35]. We also include the constraints of bottom quarks produced in $Z$ decays, $R_{b}$, which is calculated following the formulas in $[36,37]$.

(4) The global fit to the $125 \mathrm{GeV}$ Higgs signal data. The version 2.0 of Lilith [38] is used to perform the $\chi^{2}$ calculation for the signal strengths of the $125 \mathrm{GeV}$ Higgs boson combining the LHC run-I and run-II data (up to datasets of $36 \mathrm{fb}^{-1}$ ). We pay particular attention to the surviving samples with $\chi^{2}-\chi_{\min }^{2} \leq$ 6.18 , where $\chi_{\min }^{2}$ denotes the minimum of $\chi^{2}$. These samples correspond to be within the $2 \sigma$ range in any two-dimensional plane of the model parameters when explaining the Higgs data.

(5) The exclusion limits of searches for additional Higgs bosons. We use HiggsBounds-4.3.1 $[39,40]$ to implement the exclusion constraints from the neutral and charged Higgs boson searches at LEP at 95\% confidence level.

Because the $b$-quark loop and top quark loop have destructive interference contributions to $g g \rightarrow A$ production in the type II $2 \mathrm{HDM}$, the cross section decreases with an increase of $\tan \beta$, reaches the minimum value for the moderate $\tan \beta$, and is dominated by the $b$-quark loop for enough large $\tan \beta$. In addition to $\tan \beta$ and $m_{H}$, the cross section of $g g \rightarrow H$ depends on $\sin (\beta-\alpha)$. We employ SusHi to compute the cross sections for $H$ and $A$ in the gluon fusion and $b \bar{b}$-associated production at nextto-next-to leading order in quantum chromodynamics [41]. The cross sections of $H$ via the vector boson fusion process are deduced from results of the LHC Higgs Cross Section Working Group [42]. We employ $2 \mathrm{HDMC}$ to calculate the branching ratios of the various decay modes of $H$ and $A$. The searches for the additional Higgs bosons considered by us are listed in Tables I and II. The LHC searches for $H^{ \pm}$ can not impose any constraints on the model for $m_{H^{ \pm}}>500 \mathrm{GeV}$ and $1 \leq \tan \beta \leq 25$ [43]. Therefore, we do not consider the constraints from the searches for the heavily charged Higgs bosons.

\section{B. Results and discussions}

In Fig. 1, we show $\sin (\beta-\alpha)$ and $\tan \beta$ allowed by the $125 \mathrm{GeV}$ Higgs signal data at the LHC. From Fig. 1, we see that $\tan \beta$ and $\sin (\beta-\alpha)$ have strong correlation due to the constraints of the $125 \mathrm{GeV}$ Higgs data, especially for the case of the wrong sign Yukawa coupling. The wrong sign Yukawa coupling can be achieved only for $\sin (\beta-\alpha)>0$, and $\tan \beta$ is restricted to be in a very narrow range for a given $\sin (\beta-\alpha)$. For the case of the SM-like coupling, $\sin (\beta-\alpha)$ is required to be in two very narrow ranges of $-1.0 \sim-0.99993$ and $0.994 \sim 1.0 . \tan \beta$ is allowed to be as low as 1.0, and its upper bound increases with $|\sin (\beta-\alpha)|$ in the case of the SM-like Higgs coupling.

Now, we examine the parameter space of 2HDMIID using the exclusion limits of searches for additional Higgs bosons at the LHC. In the 2HDMIID, we take the heavy $\mathrm{CP}$-even Higgs boson $\mathrm{H}$ as the only portal between DM and SM sectors, and the decay $H \rightarrow S S$ opens for $2 m_{S}<m_{H}$. The decay mode possibly affects the allowed parameter space, but the constraints of the DM observables have to be simultaneously considered. Here we temporarily assume $2 m_{S}>m_{H}$, and close the $H \rightarrow S S$ decay mode. In the next section, the effects of $H \rightarrow S S$ will be considered by combining the DM observables.

In Fig. 2, we project the surviving samples with the SMlike coupling on the planes of $m_{H}$ versus $\tan \beta$ and $m_{H}$ versus $|\sin (\beta-\alpha)|$ after imposing the constraints of preLHC (denoting theoretical constraints, electroweak precision data, the flavor observables, $R_{b}$, and the exclusion limits from searches for Higgs bosons at the LEP), the $125 \mathrm{GeV}$ Higgs boson signal data, and the searches for additional Higgs bosons at the LHC. Note that in the region of $\sin (\beta-\alpha)<0$, the signal data of the $125 \mathrm{GeV}$ Higgs boson require $\sin (\beta-\alpha)$ to nearly equal -1.0 , as shown in right panel of Fig. 1. For such a case, the couplings of $H$ and $A$ are almost the same as those in the case of $\sin (\beta-\alpha)=1.0$. Therefore, we do not distinguish the sign of $\sin (\beta-\alpha)$ when discussing the constraints on $m_{H}$ and $m_{A}$ from the LHC direct searches. 
TABLE I. The upper limits at $95 \%$ C.L. on the production cross section times branching ratio of $\tau^{+} \tau^{-}, \mu^{+} \mu^{-}, \gamma \gamma, W W$, and $Z Z$ considered in the $H$ and $A$ searches at the LHC.

\begin{tabular}{|c|c|c|c|}
\hline Channel & Experiment & Mass range $[\mathrm{GeV}]$ & Luminosity \\
\hline$g g / b \bar{b} \rightarrow H / A \rightarrow \tau^{+} \tau^{-}$ & ATLAS $8 \mathrm{TeV}$ [44] & $90-1000$ & $19.5-20.3 \mathrm{fb}^{-1}$ \\
\hline$g g / b \bar{b} \rightarrow H / A \rightarrow \tau^{+} \tau^{-}$ & CMS $8 \mathrm{TeV}$ [45] & $90-1000$ & $19.7 \mathrm{fb}^{-1}$ \\
\hline$g g / b \bar{b} \rightarrow H / A \rightarrow \tau^{+} \tau^{-}$ & CMS $13 \mathrm{TeV}$ [46] & $90-3200$ & $12.9 \mathrm{fb}^{-1}$ \\
\hline$g g / b \bar{b} \rightarrow H / A \rightarrow \tau^{+} \tau^{-}$ & CMS $13 \mathrm{TeV}$ [47] & $200-2250$ & $36.1 \mathrm{fb}^{-1}$ \\
\hline$b \bar{b} \rightarrow H / A \rightarrow \tau^{+} \tau^{-}$ & CMS $8 \mathrm{TeV}$ [48] & $25-80$ & $19.7 \mathrm{fb}^{-1}$ \\
\hline$g g / b \bar{b} \rightarrow H / A \rightarrow \tau^{+} \tau^{-}$ & ATLAS $13 \mathrm{TeV}$ [49] & $200-2500$ & $139 \mathrm{fb}^{-1}$ \\
\hline$b \bar{b} \rightarrow H / A \rightarrow \mu^{+} \mu^{-}$ & CMS $8 \mathrm{TeV}[50]$ & $25-60$ & $19.7 \mathrm{fb}^{-1}$ \\
\hline$p p \rightarrow H / A \rightarrow \gamma \gamma$ & ATLAS $13 \mathrm{TeV}$ [51] & $200-2400$ & $15.4 \mathrm{fb}^{-1}$ \\
\hline$g g \rightarrow H / A \rightarrow \gamma \gamma$ & CMS $8+13 \mathrm{TeV}[52]$ & $500-4000$ & $12.9 \mathrm{fb}^{-1}$ \\
\hline$g g \rightarrow H / A \rightarrow \gamma \gamma+t \bar{t} H / A(H / A \rightarrow \gamma \gamma)$ & CMS $8 \mathrm{TeV}$ [53] & $80-110$ & $19.7 \mathrm{fb}^{-1}$ \\
\hline$g g \rightarrow H / A \rightarrow \gamma \gamma+t \bar{t} H / A(H / A \rightarrow \gamma \gamma)$ & CMS $13 \mathrm{TeV}$ [53] & $70-110$ & $35.9 \mathrm{fb}^{-1}$ \\
\hline$V V \rightarrow H \rightarrow \gamma \gamma+V H(H \rightarrow \gamma \gamma)$ & CMS $8 \mathrm{TeV}$ [53] & $80-110$ & $19.7 \mathrm{fb}^{-1}$ \\
\hline$V V \rightarrow H \rightarrow \gamma \gamma+V H(H \rightarrow \gamma \gamma)$ & CMS $13 \mathrm{TeV}[53]$ & $70-110$ & $35.9 \mathrm{fb}^{-1}$ \\
\hline$g g / V V \rightarrow H \rightarrow W^{+} W^{-}$ & ATLAS $8 \mathrm{TeV}$ [54] & $300-1500$ & $20.3 \mathrm{fb}^{-1}$ \\
\hline$g g / V V \rightarrow H \rightarrow W^{+} W^{-}(\ell \nu \ell \nu)$ & ATLAS $13 \mathrm{TeV}$ [55] & $300-3000$ & $13.2 \mathrm{fb}^{-1}$ \\
\hline$g g \rightarrow H \rightarrow W^{+} W^{-}(\ell \nu q q)$ & ATLAS $13 \mathrm{TeV}$ [56] & $500-3000$ & $13.2 \mathrm{fb}^{-1}$ \\
\hline$g g / V V \rightarrow H \rightarrow W^{+} W^{-}(\ell \nu q q)$ & ATLAS $13 \mathrm{TeV}$ [57] & $200-3000$ & $36.1 \mathrm{fb}^{-1}$ \\
\hline$g g / V V \rightarrow H \rightarrow W^{+} W^{-}(e \nu \mu \nu)$ & ATLAS $13 \mathrm{TeV}$ [58] & $200-3000$ & $36.1 \mathrm{fb}^{-1}$ \\
\hline$g g / V V \rightarrow H \rightarrow W^{+} W^{-}$ & CMS $13 \mathrm{TeV}[59]$ & $200-3000$ & $35.9 \mathrm{fb}^{-1}$ \\
\hline$g g / V V \rightarrow H \rightarrow Z Z$ & ATLAS $8 \mathrm{TeV}$ [60] & $160-1000$ & $20.3 \mathrm{fb}^{-1}$ \\
\hline$g g \rightarrow H \rightarrow Z Z(\ell \ell \nu \nu)$ & ATLAS $13 \mathrm{TeV}$ [61] & $300-1000$ & $13.3 \mathrm{fb}^{-1}$ \\
\hline$g g \rightarrow H \rightarrow Z Z(\nu \nu q q)$ & ATLAS $13 \mathrm{TeV}$ [62] & $300-3000$ & $13.2 \mathrm{fb}^{-1}$ \\
\hline$g g / V V \rightarrow H \rightarrow Z Z(\ell \ell q q)$ & ATLAS $13 \mathrm{TeV}$ [62] & $300-3000$ & $13.2 \mathrm{fb}^{-1}$ \\
\hline$g g / V V \rightarrow H \rightarrow Z Z(\ell \ell \ell \ell)$ & ATLAS $13 \mathrm{TeV}$ [63] & $200-3000$ & $14.8 \mathrm{fb}^{-1}$ \\
\hline$g g / V V \rightarrow H \rightarrow Z Z(\ell \ell \ell \ell+\ell \ell \nu \nu)$ & ATLAS $13 \mathrm{TeV}$ [64] & 200-2000 & $36.1 \mathrm{fb}^{-1}$ \\
\hline$g g / V V \rightarrow H \rightarrow Z Z(\nu \nu q q+\ell \ell q q)$ & ATLAS $13 \mathrm{TeV}$ [65] & $300-5000$ & $36.1 \mathrm{fb}^{-1}$ \\
\hline
\end{tabular}

From Fig. 2, we find the joint constraints of $H / A \rightarrow \tau^{+} \tau^{-}, A \rightarrow H Z, H \rightarrow W W, Z Z, \gamma \gamma$, and $H \rightarrow h h$ exclude the whole region of $m_{H}<360 \mathrm{GeV}$. The $H / A \rightarrow$ $\tau^{+} \tau^{-}$channels impose an upper bound on $\tan \beta$ in the whole range of $m_{H}$, and allow $m_{H}$ to vary from $150 \mathrm{GeV}$ to $800 \mathrm{GeV}$ for appropriate values of $\tan \beta$ and $\sin (\beta-\alpha)$. The $A \rightarrow H Z$ channel does not constrain the parameter space of $m_{H}>360 \mathrm{GeV}$ since the branching ratio of $A \rightarrow H Z$ rapidly decreases with an increase of $m_{H}$. The limits of the $A \rightarrow H Z$ channel can be relaxed by a small $|\sin (\beta-\alpha)|$ which suppresses the $A H Z$ coupling.

The $H \rightarrow W W, Z Z, \gamma \gamma, h h$, and $A \rightarrow h Z$ channels impose strong constraints on the regions with small values of $|\sin (\beta-\alpha)|$ and $\tan \beta$ since the couplings of $H W W$, $H Z Z$, $H h h$, and $A h Z$ increase with the decrease of $|\sin (\beta-\alpha)|$, and $\sigma(g g \rightarrow H / A)$ is enhanced by the top quark loop for a small $\tan \beta$. In addition, Fig. 1 shows that the $125 \mathrm{GeV}$ Higgs boson signal data favor a small $\tan \beta$ for a small $|\sin (\beta-\alpha)|$ in the case of the SM-like coupling. With an increase of $m_{H}$, the $H \rightarrow t \bar{t}$ channel opens and enhances the total width of $H$ sizably, so that the constraints from the $H \rightarrow W W, Z Z, \gamma \gamma$, and $h h$ channels are relaxed. Different from the other channels, the $A h Z$ channel gives the constraints on the region with a large $m_{H}$. This is because the width of $A \rightarrow H Z$ decreases with an increase of $m_{H}$, and thus $\operatorname{Br}(A \rightarrow h Z)$ increases with $m_{H}$.

It is noted that some allowed samples lie in the region of $\tan \beta<1.5$ and the other region is empty for $m_{H}>$ $700 \mathrm{GeV}$ in the left panel of Fig. 2. The main reason is from the theoretical constraints. The vacuum stability requires that

$$
\begin{aligned}
& \lambda_{1}>0, \quad \lambda_{2}>0, \quad \lambda_{3}>-\sqrt{\lambda_{1} \lambda_{2}}, \\
& \lambda_{3}+\lambda_{4}-\left|\lambda_{5}\right|>-\sqrt{\lambda_{1} \lambda_{2}} .
\end{aligned}
$$

Here, we focus on the case of the SM-like coupling, and $\cos (\beta-\alpha)$ is very small. Therefore, we can approximately obtain the following relations [88]: 
TABLE II. The upper limits at 95\% C.L. on the production cross section times branching ratio for the channels of Higgs-pair and a Higgs production in association with $Z$ at the LHC.

\begin{tabular}{|c|c|c|c|}
\hline Channel & Experiment & Mass range $[\mathrm{GeV}]$ & Luminosity \\
\hline$g g \rightarrow H \rightarrow h h \rightarrow(\gamma \gamma)(b \bar{b})$ & CMS $8 \mathrm{TeV}$ [66] & $250-1100$ & $19.7 \mathrm{fb}^{-1}$ \\
\hline$g g \rightarrow H \rightarrow h h \rightarrow(b \bar{b})(b \bar{b})$ & CMS $8 \mathrm{TeV}$ [67] & $270-1100$ & $17.9 \mathrm{fb}^{-1}$ \\
\hline$g g \rightarrow H \rightarrow h h \rightarrow(b \bar{b})\left(\tau^{+} \tau^{-}\right)$ & CMS $8 \mathrm{TeV}$ [68] & $260-350$ & $19.7 \mathrm{fb}^{-1}$ \\
\hline$g g \rightarrow H \rightarrow h h \rightarrow b \bar{b} b \bar{b}$ & ATLAS $13 \mathrm{TeV}$ [69] & $300-3000$ & $13.3 \mathrm{fb}^{-1}$ \\
\hline$g g \rightarrow H \rightarrow h h \rightarrow b \bar{b} b \bar{b}$ & CMS $13 \mathrm{TeV}$ [70] & $750-3000$ & $35.9 \mathrm{fb}^{-1}$ \\
\hline$g g \rightarrow H \rightarrow h h \rightarrow(b \bar{b})\left(\tau^{+} \tau^{-}\right)$ & CMS $13 \mathrm{TeV}$ [71] & $250-900$ & $35.9 \mathrm{fb}^{-1}$ \\
\hline$p p \rightarrow H \rightarrow h h$ & CMS $13 \mathrm{TeV}$ [72] & $250-3000$ & $35.9 \mathrm{fb}^{-1}$ \\
\hline$g g \rightarrow H \rightarrow h h \rightarrow b \bar{b} Z Z$ & CMS $13 \mathrm{TeV}$ [73] & $260-1000$ & $35.9 \mathrm{fb}^{-1}$ \\
\hline$g g \rightarrow H \rightarrow h h \rightarrow b \bar{b} \tau^{+} \tau^{-}$ & CMS $13 \mathrm{TeV}[74]$ & $1000-3000$ & $139 \mathrm{fb}^{-1}$ \\
\hline$g g \rightarrow A \rightarrow h Z \rightarrow\left(\tau^{+} \tau^{-}\right)(\ell \ell)$ & CMS $8 \mathrm{TeV}[68]$ & $220-350$ & $19.7 \mathrm{fb}^{-1}$ \\
\hline$g g \rightarrow A \rightarrow h Z \rightarrow(b \bar{b})(\ell \ell)$ & CMS $8 \mathrm{TeV}$ [75] & $225-600$ & $19.7 \mathrm{fb}^{-1}$ \\
\hline$g g \rightarrow A \rightarrow h Z \rightarrow\left(\tau^{+} \tau^{-}\right) Z$ & ATLAS $8 \mathrm{TeV}$ [76] & $220-1000$ & $20.3 \mathrm{fb}^{-1}$ \\
\hline$g g \rightarrow A \rightarrow h Z \rightarrow(b \bar{b}) Z$ & ATLAS $8 \mathrm{TeV}$ [76] & $220-1000$ & $20.3 \mathrm{fb}^{-1}$ \\
\hline$g g / b \bar{b} \rightarrow A \rightarrow h Z \rightarrow(b \bar{b}) Z$ & ATLAS $13 \mathrm{TeV}$ [77] & $200-2000$ & $36.1 \mathrm{fb}^{-1}$ \\
\hline$g g / b \bar{b} \rightarrow A \rightarrow h Z \rightarrow(b \bar{b}) Z$ & CMS $13 \mathrm{TeV}$ [78] & $225-1000$ & $35.9 \mathrm{fb}^{-1}$ \\
\hline$g g \rightarrow A \rightarrow h Z \rightarrow\left(\tau^{+} \tau^{-}\right)(\ell \ell)$ & CMS $13 \mathrm{TeV}$ [79] & $220-400$ & $35.9 \mathrm{fb}^{-1}$ \\
\hline$g g \rightarrow h \rightarrow A A \rightarrow \tau^{+} \tau^{-} \tau^{+} \tau^{-}$ & ATLAS $8 \mathrm{TeV}[80]$ & $4-50$ & $20.3 \mathrm{fb}^{-1}$ \\
\hline$p p \rightarrow h \rightarrow A A \rightarrow \tau^{+} \tau^{-} \tau^{+} \tau^{-}$ & CMS $8 \mathrm{TeV}$ [81] & $5-15$ & $19.7 \mathrm{fb}^{-1}$ \\
\hline$p p \rightarrow h \rightarrow A A \rightarrow\left(\mu^{+} \mu^{-}\right)(b \bar{b})$ & CMS $8 \mathrm{TeV}$ [81] & $25-62.5$ & $19.7 \mathrm{fb}^{-1}$ \\
\hline$p p \rightarrow h \rightarrow A A \rightarrow\left(\mu^{+} \mu^{-}\right)\left(\tau^{+} \tau^{-}\right)$ & CMS $8 \mathrm{TeV}[81]$ & $15-62.5$ & $19.7 \mathrm{fb}^{-1}$ \\
\hline$p p \rightarrow h \rightarrow A A \rightarrow(b \bar{b})\left(\tau^{+} \tau^{-}\right)$ & CMS $13 \mathrm{TeV}$ [82] & $15-60$ & $35.9 \mathrm{fb}^{-1}$ \\
\hline$p p \rightarrow h \rightarrow A A \rightarrow \tau^{+} \tau^{-} \tau^{+} \tau^{-}$ & CMS $13 \mathrm{TeV}$ [83] & $4-15$ & $35.9 \mathrm{fb}^{-1}$ \\
\hline$p p \rightarrow h \rightarrow A A \rightarrow \mu^{+} \mu^{-} \tau^{+} \tau^{-}$ & CMS $13 \mathrm{TeV}[84]$ & $3.6-21$ & $35.9 \mathrm{fb}^{-1}$ \\
\hline$g g \rightarrow A(H) \rightarrow H(A) Z \rightarrow(b \bar{b})(\ell \ell)$ & CMS $8 \mathrm{TeV}[85]$ & $40-1000$ & $19.8 \mathrm{fb}^{-1}$ \\
\hline$g g \rightarrow A(H) \rightarrow H(A) Z \rightarrow\left(\tau^{+} \tau^{-}\right)(\ell \ell)$ & CMS $8 \mathrm{TeV}$ [85] & $20-1000$ & $19.8 \mathrm{fb}^{-1}$ \\
\hline$g g / b \bar{b} \rightarrow A(H) \rightarrow H(A) Z \rightarrow(b \bar{b})(\ell \ell)$ & ATLAS $13 \mathrm{TeV}$ [86] & $130-800$ & $36.1 \mathrm{fb}^{-1}$ \\
\hline$g g \rightarrow A(H) \rightarrow H(A) Z \rightarrow(b \bar{b})(\ell \ell)$ & CMS $13 \mathrm{TeV}$ [87] & $30-1000$ & $35.9 \mathrm{fb}^{-1}$ \\
\hline
\end{tabular}
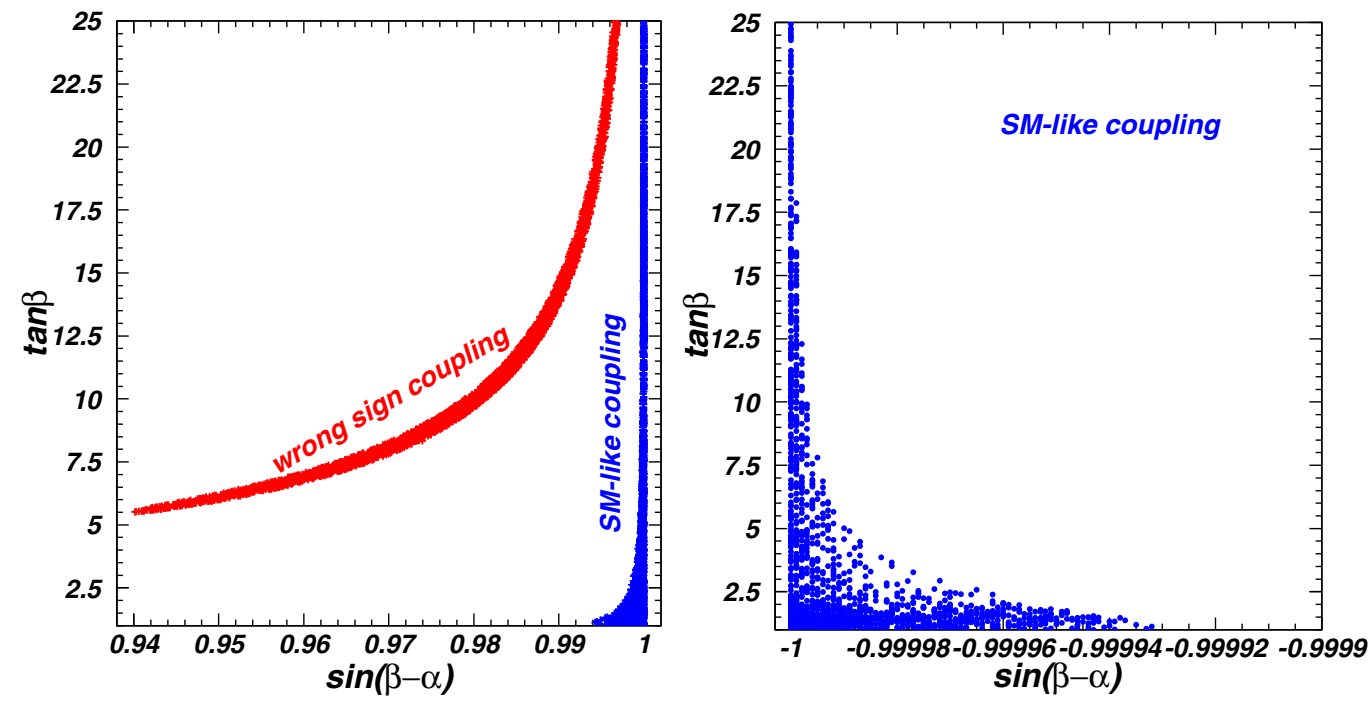

FIG. 1. Scatter plots of $\sin (\beta-\alpha)$ and $\tan \beta$ satisfying the constraints of the $125 \mathrm{GeV}$ Higgs boson signal data. 

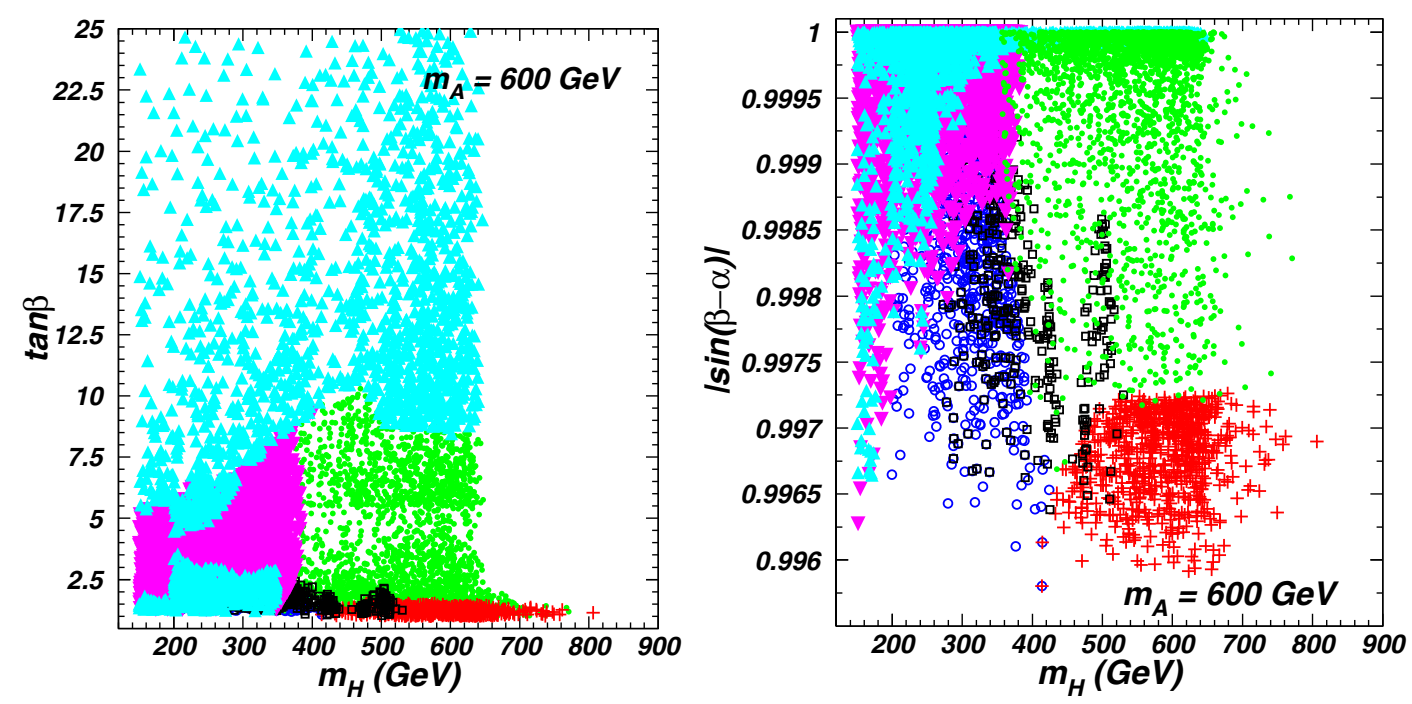

FIG. 2. The surviving samples with the SM-like coupling projected on the planes of $m_{H}$ versus $\tan \beta$ and $m_{H}$ versus $|\sin (\beta-\alpha)|$. All of the samples are allowed by the constraints of pre-LHC and the $125 \mathrm{GeV}$ Higgs boson signal data. The triangles (sky blue), circles (royal blue), squares (black), inverted triangles (purple), and pluses (red) are respectively excluded by the $H / A \rightarrow \tau^{+} \tau^{-}$, $H \rightarrow W W, Z Z, \gamma \gamma, H \rightarrow h h, A \rightarrow H Z$, and $A \rightarrow h Z$ channels at the LHC. The bullets (green) are allowed by various LHC direct searches.

$v^{2} \lambda_{1}=m_{h}^{2}-\frac{t_{\beta}^{3}\left(m_{12}^{2}-m_{H}^{2} s_{\beta} c_{\beta}\right)}{s_{\beta}^{2}}$,

$v^{2} \lambda_{2}=m_{h}^{2}-\frac{\left(m_{12}^{2}-m_{H}^{2} s_{\beta} c_{\beta}\right)}{t_{\beta} s_{\beta}^{2}}$,

$v^{2} \lambda_{3}=m_{h}^{2}+2 m_{H^{ \pm}}^{2}-2 m_{H}^{2}-\frac{t_{\beta}\left(m_{12}^{2}-m_{H}^{2} s_{\beta} c_{\beta}\right)}{s_{\beta}^{2}}$,

$v^{2} \lambda_{4}=m_{A}^{2}-2 m_{H^{ \pm}}^{2}+m_{H}^{2}+\frac{t_{\beta}\left(m_{12}^{2}-m_{H}^{2} s_{\beta} c_{\beta}\right)}{s_{\beta}^{2}}$,

$v^{2} \lambda_{5}=m_{H}^{2}-m_{A}^{2}+\frac{t_{\beta}\left(m_{12}^{2}-m_{H}^{2} s_{\beta} c_{\beta}\right)}{s_{\beta}^{2}}$,

with $\quad t_{\beta} \equiv \tan \beta, \quad s_{\beta} \equiv \sin \beta, \quad$ and $\quad c_{\beta} \equiv \cos \beta$. If $m_{12}^{2}-m_{H}^{2} s_{\beta} c_{\beta} \rightarrow 0$, the first two requirements in Eq. (11) are simultaneously satisfied, and the last condition will require that

$$
m_{h}^{2}+m_{A}^{2}-m_{H}^{2}>0 .
$$

However, the relation of Eq. (13) is not satisfied for $m_{h}=125 \mathrm{GeV}, m_{A}=600 \mathrm{GeV}$, and $m_{H}>700 \mathrm{GeV}$. Therefore, for a large $m_{H}, m_{12}^{2}-m_{H}^{2} s_{\beta} c_{\beta}$ is not allowed to approach 0 . The first expression of Eq. (12) shows that the term of $m_{12}^{2}-m_{H}^{2} s_{\beta} c_{\beta}$ is enhanced by a factor of $t_{\beta}^{3}$. Therefore, the vacuum stability and perturbativity favor a small $\tan \beta$ for a large $m_{H}$.

\section{THE DARK MATTER OBSERVABLES}

We use FeynRules [89] to generate the model file, which is called by micromEGAs [90] to calculate the relic density. In our scenario, the elastic scattering of $S$ on a nucleon receives the contributions of the process with a $t$-channel exchange of $H$, and the spin-independent cross section between DM and nucleons is given by [91]

$$
\sigma_{p(n)}=\frac{\mu_{p(n)}^{2}}{4 \pi m_{S}^{2}}\left[f^{p(n)}\right]^{2},
$$

where $\mu_{p(n)}=\frac{m_{S} m_{p(n)}}{m_{S}+m_{p(n)}}$, and

$f^{p(n)}=\sum_{q=u, d, s} f_{q}^{p(n)} \mathcal{C}_{S q} \frac{m_{p(n)}}{m_{q}}+\frac{2}{27} f_{g}^{p(n)} \sum_{q=c, b, t} \mathcal{C}_{S q} \frac{m_{p(n)}}{m_{q}}$,

with $\mathcal{C}_{S q}=\frac{\lambda_{H}}{m_{H}^{2}} m_{q} y_{H}^{q}$. The values of the form factors $f_{q}^{p, n}$ and $f_{g}^{p, n}$ are extracted from micromeGAs [90].

The Planck Collaboration reported the relic density of cold DM in the universe, $\Omega_{c} h^{2}=0.1198 \pm 0.0015$ [92]. The XENON1T Collaboration reported stringent upper bounds of the spin-independent DM-nucleon cross section [93]. The Fermi-LAT Collaboration searches for the DM annihilation from dwarf spheroidal satellite galaxies gave the upper limits on the averaged cross sections of the DM annihilation to $e^{+} e^{-}, \mu^{+} \mu^{-}, \tau^{+} \tau^{-}, u \bar{u}, b \bar{b}$, and $W W$ [94].

In Fig. 3, we project the surviving samples on the planes of $\lambda_{H}$ versus $m_{S}, m_{H}$ versus $m_{S}$, and $\sigma_{p}$ versus $m_{S}$ after 

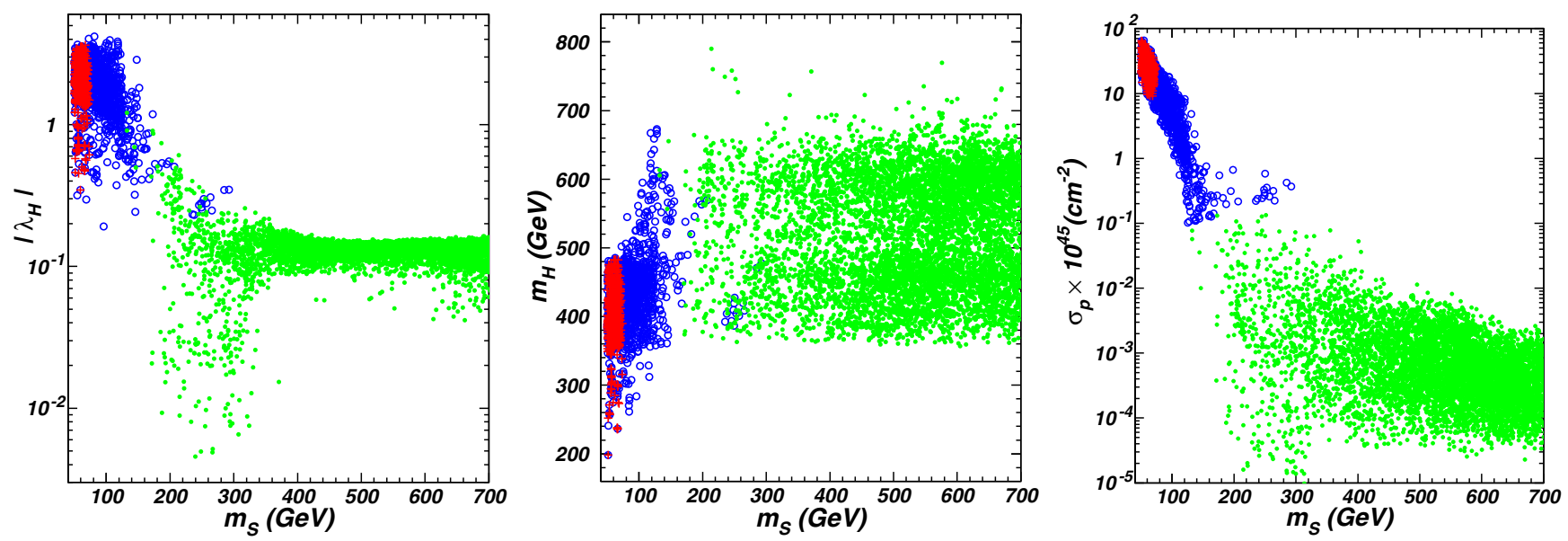

FIG. 3. The surviving samples projected on the planes of $m_{S}$ versus $\lambda_{H}, m_{S}$ versus $m_{H}$, and $m_{S}$ versus $\sigma_{p}$. All of the samples are allowed by the constraints of "pre-LHC", the LHC Higgs data, and the relic density. The circles (royal blue) and pluses (red) are, respectively, excluded by the experimental data of the XENON1T and Fermi-LAT Collaborations, while the bullets (green) are allowed.

imposing the constraints of "pre-LHC", the Higgs data at the LHC, the relic density, XENON1T, and Fermi-LAT. The middle panel shows that the $H \rightarrow S S$ decay weakens the constraints of the LHC Higgs data compared to Fig. 2. For example, $m_{H}$ is allowed to be as low as $200 \mathrm{GeV}$ for a light DM. However, the upper bounds of the XENON1T and Fermi-LAT Collaborations exclude $m_{S}<130 \mathrm{GeV}$ and $m_{H}<360 \mathrm{GeV}$. In order to obtain the correct relic density, $\left|\lambda_{H}\right|$ is favored to increase with the decrease of $m_{S}$. Thus, when $m_{S}$ is small, a large $\left|\lambda_{H}\right|$ can enhance the spin-independent DM-nucleon cross section and the averaged cross sections of the DM annihilation to the SM particles, leading that $m_{S}<130 \mathrm{GeV}$ and $m_{S}<75 \mathrm{GeV}$ are, respectively, excluded by the experimental data of the XENON1T and Fermi-LAT Collaborations. For $180 \mathrm{GeV}<m_{S}<340 \mathrm{GeV},\left|\lambda_{H}\right|$ can be allowed to be smaller than 0.01 because of the resonant contribution at $2 m_{S} \sim m_{H}$.

\section{ELECTROWEAK PHASE TRANSITION AND GRAVITATIONAL WAVE}

The phase transition can basically proceed in two different ways. In a first order phase transition, at the critical temperature $T_{C}$, the two degenerate minima will be at different points in field space, typically with a potential barrier in between. For a second order (crossover) transition, the broken and symmetric minimum are not degenerate until they are at the same point in field space. In this paper we focus on the SFOEWPT, which is required by a successful explanation of the observed BAU and can produce primordial GW signals.

\section{A. The thermal effective potential}

In order to examine the electroweak phase transition (EWPT), we first take $h_{1}, h_{2}$, and $S_{1}$ as the field configurations, and obtain the field dependent masses of the scalars $\left(h, H, A, H^{ \pm}, S\right)$, the Goldstone boson $\left(G, G^{ \pm}\right)$, the gauge boson, and fermions. The masses of scalars are given:

$$
\begin{gathered}
\hat{m}_{h, H, S}^{2}=\operatorname{eigenvalues}\left(\widehat{\mathcal{M}_{P}^{2}}\right), \\
\hat{m}_{G, A}^{2}=\operatorname{eigenvalues}\left(\widehat{\mathcal{M}_{A}^{2}}\right), \\
\hat{m}_{G^{ \pm}, H^{ \pm}}^{2}=\operatorname{eigenvalues}\left(\widehat{\mathcal{M}_{C}^{2}}\right),
\end{gathered}
$$

$$
\begin{aligned}
\widehat{\mathcal{M}_{P 11}^{2}}= & \frac{3 \lambda_{1}}{2} h_{1}^{2}+\frac{\lambda_{345}}{2} h_{2}^{2}+m_{12}^{2} t_{\beta}-\frac{\lambda_{1}}{2} v^{2} c_{\beta}^{2} \\
& -\frac{\lambda_{345}}{2} v^{2} s_{\beta}^{2}+\frac{\kappa_{1}}{2} S_{1}^{2}, \\
\widehat{\mathcal{M}_{P 22}^{2}}= & \frac{3 \lambda_{2}}{2} h_{2}^{2}+\frac{\lambda_{345}}{2} h_{1}^{2}+\frac{m_{12}^{2}}{t_{\beta}}-\frac{\lambda_{2}}{2} v^{2} s_{\beta}^{2}-\frac{\lambda_{345}}{2} v^{2} c_{\beta}^{2}+\frac{\kappa_{2}}{2} S_{1}^{2}, \\
\widehat{\mathcal{M}_{P 33}^{2}}= & m_{S}^{2}+\frac{\kappa_{1}}{2} h_{1}^{2}+\frac{\kappa_{2}}{2} h_{2}^{2}+\frac{\lambda_{S}}{2} S_{1}^{2}-\frac{\kappa_{1}}{2} v^{2} c_{\beta}^{2}-\frac{\kappa_{2}}{2} v^{2} s_{\beta}^{2}, \\
\widehat{\mathcal{M}_{P 12}^{2}}= & \widehat{\mathcal{M}_{P 21}^{2}}=\lambda_{345} h_{1} h_{2}-m_{12}^{2}, \\
\widehat{\mathcal{M}_{P 13}^{2}}= & \widehat{\mathcal{M}_{P 31}^{2}}=\kappa_{1} h_{1} S_{1}, \\
\widehat{\mathcal{M}_{P 23}^{2}}= & \widehat{\mathcal{M}_{P 32}^{2}}=\kappa_{2} h_{2} S_{1}, \\
\widehat{\mathcal{M}_{A 11}^{2}}= & \frac{\lambda_{1}}{2} h_{1}^{2}+m_{12}^{2} t_{\beta}-\frac{\lambda_{1}}{2} v^{2} c_{\beta}^{2}-\frac{\lambda_{345}}{2} v^{2} s_{\beta}^{2} \\
& +\frac{\left(\lambda_{3}+\lambda_{4}-\lambda_{5}\right)}{2} h_{2}^{2}+\frac{\kappa_{1}}{2} S_{1}^{2}, \\
\widehat{\mathcal{M}_{A 22}^{2}}= & \frac{\lambda_{2}}{2} h_{2}^{2}+\frac{m_{12}^{2}}{t_{\beta}}-\frac{\lambda_{2}}{2} v^{2} s_{\beta}^{2}-\frac{\lambda_{345}}{2} v^{2} c_{\beta}^{2} \\
& +\frac{\left(\lambda_{3}+\lambda_{4}-\lambda_{5}\right)}{2} h_{1}^{2}+\frac{\kappa_{2}}{2} S_{1}^{2}, \\
\widehat{\mathcal{M}_{A 12}^{2}}= & \widehat{\mathcal{M}_{A 21}^{2}}=\lambda_{5} h_{1} h_{2}-m_{12}^{2},
\end{aligned}
$$


$\widehat{\mathcal{M}_{C 11}^{2}}=\frac{\lambda_{1}}{2} h_{1}^{2}+m_{12}^{2} t_{\beta}-\frac{\lambda_{1}}{2} v^{2} c_{\beta}^{2}-\frac{\lambda_{345}}{2} v^{2} s_{\beta}^{2}+\frac{\lambda_{3}}{2} h_{2}^{2}+\frac{\kappa_{1}}{2} S_{1}^{2}$,

$\widehat{\mathcal{M}_{C 22}^{2}}=\frac{\lambda_{2}}{2} h_{2}^{2}+\frac{m_{12}^{2}}{t_{\beta}}-\frac{\lambda_{2}}{2} v^{2} s_{\beta}^{2}-\frac{\lambda_{345}}{2} v^{2} c_{\beta}^{2}+\frac{\lambda_{3}}{2} h_{1}^{2}+\frac{\kappa_{2}}{2} S_{1}^{2}$,

$\widehat{\mathcal{M}_{C 12}^{2}}=\widehat{\mathcal{M}_{C 21}^{2}}=\frac{\left(\lambda_{4}+\lambda_{5}\right)}{2} h_{1} h_{2}-m_{12}^{2}$,

where $\lambda_{345}=\lambda_{3}+\lambda_{4}+\lambda_{5}$.

The masses of the gauge boson are given:

$$
\begin{aligned}
\hat{m}_{W^{ \pm}}^{2} & =\frac{1}{4} g^{2}\left(h_{1}^{2}+h_{2}^{2}\right), \\
\hat{m}_{Z}^{2} & =\frac{1}{4}\left(g^{2}+g^{2}\right)\left(h_{1}^{2}+h_{2}^{2}\right), \\
\hat{m}_{\gamma}^{2} & =0 .
\end{aligned}
$$

We neglect the contributions of light fermions, and only consider the masses of top quark and bottom quark,

$$
\hat{m}_{t}^{2}=\frac{1}{2} y_{t}^{2} h_{2}^{2} / s_{\beta}^{2}, \quad \hat{m}_{b}^{2}=\frac{1}{2} y_{b}^{2} h_{1}^{2} / c_{\beta}^{2},
$$

where $y_{t}=\frac{\sqrt{2} m_{t}}{v}$ and $y_{b}=\frac{\sqrt{2} m_{b}}{v}$.

Now, we study the effective potential with thermal correction. The thermal effective potential $V_{\text {eff }}$ in terms of the classical fields $\left(h_{1}, h_{2}, S_{1}\right)$ is composed of four parts:

$$
\begin{aligned}
V_{\text {eff }}\left(h_{1}, h_{2}, S_{1}, T\right)= & V_{0}\left(h_{1}, h_{2}, S_{1}\right)+V_{\mathrm{CW}}\left(h_{1}, h_{2}, S_{1}\right) \\
& +V_{\mathrm{CT}}\left(h_{1}, h_{2}, S_{1}\right)+V_{T}\left(h_{1}, h_{2}, S_{1}, T\right) \\
& +V_{\text {ring }}\left(h_{1}, h_{2}, S_{1}, T\right)
\end{aligned}
$$

where $V_{0}$ is the tree-level potential, $V_{\mathrm{CW}}$ is the ColemanWeinberg potential, $V_{\mathrm{CT}}$ is the counter term, $V_{T}$ is the thermal correction, and $V_{\text {ring }}$ is the resummed daisy correction. In this paper, we calculate $V_{\text {eff }}$ in the Landau gauge.

We obtain the tree-level potential $V_{0}$ in terms of the classical fields $\left(h_{1}, h_{2}, S_{1}\right)$

$$
\begin{aligned}
\mathcal{V}_{0}= & {\left[\frac{1}{2} m_{12}^{2} t_{\beta}-\frac{1}{4} \lambda_{1} v^{2} c_{\beta}^{2}-\frac{1}{4} \lambda_{345} v^{2} s_{\beta}^{2}\right] h_{1}^{2} } \\
& +\left[\frac{1}{2} m_{12}^{2} \frac{1}{t_{\beta}}-\frac{1}{4} \lambda_{2} v^{2} s_{\beta}^{2}-\frac{1}{4} \lambda_{345} v^{2} c_{\beta}^{2}\right] h_{2}^{2} \\
& +\frac{\lambda_{1}}{8} h_{1}^{4}+\frac{\lambda_{2}}{8} h_{2}^{4}-m_{12}^{2} h_{1} h_{2}+\frac{1}{4} \lambda_{345} h_{1}^{2} h_{2}^{2} \\
& +\frac{\kappa_{1}}{4} h_{1}^{2} S_{1}^{2}+\frac{\kappa_{2}}{4} h_{2}^{2} S_{1}^{2}+\frac{1}{2} m_{S}^{2} S_{1}^{2}+\frac{1}{24} \lambda_{s} S_{1}^{4} \\
& -\frac{\kappa_{1}}{4} v^{2} c_{\beta}^{2} S_{1}^{2}-\frac{\kappa_{2}}{4} v^{2} s_{\beta}^{2} S_{1}^{2} .
\end{aligned}
$$

The Coleman-Weinberg potential in the $\overline{\mathrm{MS}}$ scheme at the 1-loop level has the form [95]

$$
\begin{aligned}
V_{\mathrm{CW}}\left(h_{1}, h_{2}, S_{1}\right)= & \sum_{i}(-1)^{2 s_{i}} n_{i} \frac{\hat{m}_{i}^{4}\left(h_{1}, h_{2}, S_{1}\right)}{64 \pi^{2}} \\
& \times\left[\ln \frac{\hat{m}_{i}^{2}\left(h_{1}, h_{2}, S_{1}\right)}{Q^{2}}-C_{i}\right],
\end{aligned}
$$

where $i=h, H, A, H^{ \pm}, S, G, G^{ \pm}, W^{ \pm}, Z, t, b$, and $s_{i}$ is the spin of particle $i$. $Q$ is a renormalization scale, and we take $Q^{2}=v^{2}$. The constants $C_{i}=\frac{3}{2}$ for scalars or fermions and $C_{i}=\frac{6}{5}$ for gauge bosons. $n_{i}$ is the number of degrees of freedom, and

$$
\begin{aligned}
n_{h} & =n_{H}=n_{G}=n_{A}=1, \\
n_{H^{ \pm}} & =n_{G^{ \pm}}=2, \\
n_{W^{ \pm}} & =6, \quad n_{Z}=3, \\
n_{t} & =n_{b}=12 .
\end{aligned}
$$

With $V_{\mathrm{CW}}$ being included in the potential, the minimization conditions of scalar potential in Eq. (22) and the $C P$-even mass matrix will be shifted slightly. To maintain the minimization conditions at $T=0$, we add the so-called "counter-terms",

$$
\begin{aligned}
V_{\mathrm{CT}}= & \delta m_{1}^{2} h_{1}^{2}+\delta m_{2}^{2} h_{2}^{2}+\delta \lambda_{1} h_{1}^{4}+\delta \lambda_{12} h_{1}^{2} h_{2}^{2}+\delta \lambda_{2} h_{2}^{4} \\
& +\delta m_{0}^{2} S_{1}^{2}+\delta \kappa_{1} h_{1}^{2} S_{1}^{2}+\delta \kappa_{2} h_{2}^{2} S_{1}^{2}
\end{aligned}
$$

where the relevant coefficients are determined by

$\frac{\partial V_{\mathrm{CT}}}{\partial h_{1}}=-\frac{\partial V_{\mathrm{CW}}}{\partial h_{1}}, \quad \frac{\partial V_{\mathrm{CT}}}{\partial h_{2}}=-\frac{\partial V_{\mathrm{CW}}}{\partial h_{2}}$,

$\frac{\partial V_{\mathrm{CT}}}{\partial S_{1}}=-\frac{\partial V_{\mathrm{CW}}}{\partial S_{1}}$,

$\frac{\partial^{2} V_{\mathrm{CT}}}{\partial h_{1} \partial h_{1}}=-\frac{\partial^{2} V_{\mathrm{CW}}}{\partial h_{1} \partial h_{1}}, \quad \frac{\partial^{2} V_{\mathrm{CT}}}{\partial h_{1} \partial h_{2}}=-\frac{\partial^{2} V_{\mathrm{CW}}}{\partial h_{1} \partial h_{2}}$,

$\frac{\partial^{2} V_{\mathrm{CT}}}{\partial h_{2} \partial h_{2}}=-\frac{\partial^{2} V_{\mathrm{CW}}}{\partial h_{2} \partial h_{2}}$,

$\frac{\partial^{2} V_{\mathrm{CT}}}{\partial S_{1} \partial S_{1}}=-\frac{\partial^{2} V_{\mathrm{CW}}}{\partial S_{1} \partial S_{1}}, \quad \frac{\partial^{2} V_{\mathrm{CT}}}{\partial h_{1} \partial S_{1}}=-\frac{\partial^{2} V_{\mathrm{CW}}}{\partial h_{1} \partial S_{1}}$,

$\frac{\partial^{2} V_{\mathrm{CT}}}{\partial h_{2} \partial S_{1}}=-\frac{\partial^{2} V_{\mathrm{CW}}}{\partial h_{2} \partial S_{1}}$,

which are evaluated at the electroweak (EW) minimum of $\left\{h_{1}=v c_{\beta}, h_{2}=v s_{\beta}, S_{1}=0\right\}$ on both sides. As a result, the VEVs of $h_{1}, h_{2}, S_{1}$, and the $C P$-even mass matrix, will not be shifted.

It is a well-known problem that the second derivative of the Coleman-Weinberg potential at $T=0$ suffers from logarithmic divergences originating from the vanishing Goldstone masses. To solve the divergence problem, we take a straightforward approach of imposing an IR cutoff at 
$m_{\mathrm{IR}}^{2}=m_{h}^{2}$ for the masses of Goldstone bosons of the divergent terms, which gives a good approximation to the exact procedure of on-shell renormalization, as argued in [16].

The thermal contributions $V_{T}$ to the potential can be written as [96]

$V_{\text {th }}\left(h_{1}, h_{2}, S_{1}, T\right)=\frac{T^{4}}{2 \pi^{2}} \sum_{i} n_{i} J_{B, F}\left(\frac{\hat{m}_{i}^{2}\left(h_{1}, h_{2}, S_{1}\right)}{T^{2}}\right)$,

where $i=h, H, A, H^{ \pm}, S, G, G^{ \pm}, W^{ \pm}, Z, t, b$, and the functions $J_{B, F}$ are

$J_{B, F}(y)= \pm \int_{0}^{\infty} d x x^{2} \ln \left[1 \mp \exp \left(-\sqrt{x^{2}+y}\right)\right]$.

Finally, the thermal corrections with resummed ring diagrams are given [97,98]:

$$
\begin{aligned}
V_{\text {ring }}\left(h_{1}, h_{2}, S_{1}, T\right)= & -\frac{T}{12 \pi} \sum_{i} n_{i}\left[\left(\bar{M}_{i}^{2}\left(h_{1}, h_{2}, S_{1}, T\right)\right)^{\frac{3}{2}}\right. \\
& \left.-\left(\hat{m}_{i}^{2}\left(h_{1}, h_{2}, S_{1}, T\right)\right)^{\frac{3}{2}}\right],
\end{aligned}
$$

where $i=h, H, A, H^{ \pm}, S, G, G^{ \pm}, W_{L}^{ \pm}, Z_{L}, \gamma_{L} . W_{L}^{ \pm}, Z_{L}$, and $\gamma_{L}$ are the longitudinal gauge bosons with $n_{W_{L}^{ \pm}}=2$, $n_{Z_{L}}=n_{\gamma_{L}}=1$. The thermal Debye masses $\bar{M}_{i}^{2}\left(h_{1}, h_{2}, S_{1}, T\right)$ are the eigenvalues of the full mass matrix,

$\bar{M}_{i}^{2}\left(h_{1}, h_{2}, T\right)=$ eigenvalues $\left[\widehat{\mathcal{M}_{X}^{2}}\left(h_{1}, h_{2}\right)+\Pi_{X}(T)\right]$,

where $X=P, A, C . \Pi_{X}$ are given by

$$
\begin{aligned}
& \Pi_{P 11}=\left[\frac{9 g^{2}}{2}+\frac{3 g^{\prime 2}}{2}+\frac{6 y_{b}^{2}}{c_{\beta}^{2}}+6 \lambda_{1}+4 \lambda_{3}+2 \lambda_{4}+\kappa_{1}\right] \frac{T^{2}}{24}, \\
& \Pi_{P 22}=\left[\frac{9 g^{2}}{2}+\frac{3 g^{\prime 2}}{2}+\frac{6 y_{t}^{2}}{s_{\beta}^{2}}+6 \lambda_{2}+4 \lambda_{3}+2 \lambda_{4}+\kappa_{2}\right] \frac{T^{2}}{24}, \\
& \Pi_{P 33}=\left[4 \kappa_{1}+4 \kappa_{2}+\lambda_{S}\right] \frac{T^{2}}{24}, \\
& \Pi_{P 13}=\Pi_{P 31}=\Pi_{P 23}=\Pi_{P 32}=0 \\
& \Pi_{A 11}=\Pi_{C 11}=\Pi_{P 11}, \\
& \Pi_{A 22}=\Pi_{C 22}=\Pi_{P 22}, \\
& \Pi_{A 12}=\Pi_{A 21}=\Pi_{C 12}=\Pi_{C 21}=0 .
\end{aligned}
$$

The physical mass of the longitudinally polarized $W$ boson is

$$
\bar{M}_{W_{L}^{ \pm}}^{2}=\frac{1}{4} g^{2}\left(h_{1}^{2}+h_{2}^{2}\right)+2 g^{2} T^{2} .
$$

The physical mass of the longitudinally polarized $Z$ and $\gamma$ boson is
$\bar{M}_{Z_{L}, \gamma_{L}}^{2}=\frac{1}{8}\left(g^{2}+g^{\prime 2}\right)\left(h_{1}^{2}+h_{2}^{2}\right)+\left(g^{2}+g^{\prime 2}\right) T^{2} \pm \Delta$,

with

$$
\begin{aligned}
\Delta^{2}= & \frac{1}{64}\left(g^{2}+g^{\prime 2}\right)^{2}\left(h_{1}^{2}+h_{2}^{2}+8 T^{2}\right)^{2} \\
& -g^{2} g^{\prime 2} T^{2}\left(h_{1}^{2}+h_{2}^{2}+4 T^{2}\right) .
\end{aligned}
$$

\section{B. Calculation of electroweak phase transition and gravitational wave}

In a first order cosmological phase transition, bubbles nucleate and expand, converting the high temperature phase into the low temperature phase. The bubble nucleation rate per unit volume at finite temperature is given by [99-101]

$$
\Gamma \approx A(T) e^{-S_{E}(T)},
$$

where $A(T) \sim T^{4}$ is a prefactor and $S_{E}$ is the Euclidean action

$$
S_{E}(T)=\frac{S_{3}(T)}{T}=\int d x^{3}\left[\frac{1}{2}\left(\frac{d \phi}{d r}\right)^{2}+V(\phi, T)\right] .
$$

At the nucleation temperature $T_{n}$, the thermal tunneling probability for bubble nucleation, per horizon volume and per horizon time, is of order one, and the conventional condition is $\frac{S_{3}(T)}{T} \approx 140$. The bubbles nucleated within one Hubble patch proceed to expand and collide, until the entire volume is filled with the true vacuum.

There are two key parameters characterizing the dynamics of the EWPT: $\beta$ and $\alpha$. $\beta$ describes, roughly, the inverse time duration of the strong first order phase transition,

$$
\frac{\beta}{H_{n}}=\left.T \frac{d\left(S_{3}(T) / T\right)}{d T}\right|_{T=T_{n}},
$$

where $H_{n}$ is the Hubble parameter at the bubble nucleation temperature $T_{n}$. $\alpha$ is defined as the vacuum energy released from the phase transition normalized by the total radiation energy density $\rho_{R}$ at $T_{n}$,

$$
\alpha=\frac{\Delta \rho}{\rho_{R}}=\frac{\Delta \rho}{\pi^{2} g_{*} T_{n}^{4} / 30},
$$

where $g_{*}$ is the effective number of relativistic degrees of freedom. We use the numerical packages CosmoTransitions [102] and PhaseTracer [103] to analyze the phase transition and compute quantities related to cosmological phase transition.

In a radiation dominated universe, there are three sources of GW production at an EWPT: bubble collisions (in which the localized energy density generates a quadrupole 
contribution to the stress-energy tensor, which in turn gives rise to the $\mathrm{GW}$ ), sound waves in the plasma, and magnetohydrodynamic (MHD) turbulence. The total resultant energy density spectrum can be approximately given as

$$
\Omega_{\mathrm{GW}} h^{2} \simeq \Omega_{\mathrm{col}} h^{2}+\Omega_{\mathrm{sw}} h^{2}+\Omega_{\mathrm{turb}} h^{2} .
$$

Recent studies show that the energy deposited in the bubble walls is negligible, despite the possibility that the bubble walls can run away in some circumstances [104]. Therefore, although a bubble wall can reach relativistic speed, its contribution to the GW can generally be neglected $[105,106]$. Therefore, in the following discussions we do not include the contribution from bubble collision $\Omega_{\mathrm{col}}$.

The GW spectrum from the sound waves can be obtained by fitting to the result of numerical simulations [107],

$$
\begin{aligned}
\Omega_{\mathrm{sw}} h^{2}= & 2.65 \times 10^{-6}\left(\frac{H_{n}}{\beta}\right)\left(\frac{\kappa_{v} \alpha}{1+\alpha}\right)^{2}\left(\frac{100}{g_{*}}\right)^{1 / 3} v_{w} \\
& \times\left(\frac{f}{f_{\mathrm{sw}}}\right)^{3}\left(\frac{7}{4+3\left(f / f_{\mathrm{sw}}\right)^{2}}\right)^{7 / 2},
\end{aligned}
$$

where $f_{\mathrm{sw}}$ is the present peak frequency of the spectrum

$f_{\mathrm{sw}}=1.9 \times 10^{-5} \frac{1}{v_{w}}\left(\frac{\beta}{H_{n}}\right)\left(\frac{T_{n}}{100 \mathrm{GeV}}\right)\left(\frac{g_{*}}{100}\right)^{1 / 6} \mathrm{~Hz}$.

$v_{w}$ is the wall velocity and the factor $\kappa_{v}$ is the fraction of latent heat transformed into the kinetic energy of the fluid. $\kappa_{v}$ and $v_{w}$ are difficult to compute, and involve certain assumptions regarding the dynamics of the bubble walls. On the other hand, successful electroweak baryogenesis scenarios favor lower wall velocity $v_{w} \leq 0.15-0.3$ [108], which allows the effective diffusion of particle asymmetries near the bubble wall front. In Ref. [109], however, it is pointed out that the relevant velocity for electroweak baryogenesis is not really $v_{w}$, but the relative velocity between the bubble wall and the plasma in the deflagration front. As a result, the electroweak baryogenesis is not necessarily impossible even in the case with large $v_{w}$. Therefore, in this paper we take two different cases of $v_{w}$ and $\kappa_{v}$ [110]:

(i) For small wall velocity: $v_{w}=0.3$ and

$$
\kappa_{v} \simeq v_{w}^{6 / 5} \frac{6.9 \alpha}{1.36-0.037 \sqrt{\alpha}+\alpha} .
$$

(ii) For very large wall velocity: $v_{w}=0.9$ and

$$
\kappa_{v} \simeq \frac{\alpha}{0.73+0.083 \sqrt{\alpha}+\alpha} .
$$

Considering Kolmogorov-type turbulence as proposed in Ref. [111], the GW spectrum from the MHD turbulence has the form $[112,113]$

$$
\begin{aligned}
\Omega_{\text {turb }} h^{2}= & 3.35 \times 10^{-4}\left(\frac{H_{n}}{\beta}\right)\left(\frac{\kappa_{\text {turb }} \alpha}{1+\alpha}\right)^{3 / 2}\left(\frac{100}{g_{*}}\right)^{1 / 3} v_{w} \\
& \times \frac{\left(f / f_{\text {turb }}\right)^{3}}{\left[1+\left(f / f_{\text {turb }}\right)\right]^{11 / 3}\left(1+8 \pi f / h_{n}\right)},
\end{aligned}
$$

with the redshifted Hubble rate at GW generation

$$
h_{n}=1.65 \times 10^{-5}\left(\frac{T_{n}}{100 \mathrm{GeV}}\right)\left(\frac{g_{*}}{100}\right)^{\frac{1}{6}} \mathrm{~Hz} .
$$

The peak frequency $f_{\text {turb }}$ is given by

$f_{\text {turb }}=2.7 \times 10^{-5} \frac{1}{v_{w}}\left(\frac{\beta}{H_{n}}\right)\left(\frac{T_{n}}{100 \mathrm{GeV}}\right)\left(\frac{g_{*}}{100}\right)^{1 / 6} \mathrm{~Hz}$.

The energy fraction transferred to the MHD turbulence $\kappa_{\text {turb }}$ can vary between $5 \%$ and $10 \%$ of $\kappa_{v}$ [107]. Here we take $\kappa_{\text {turb }}=0.1 \kappa_{v}$.

For both sound wave and turbulence contributions as shown in Eqs. (43) and (46), the amplitudes of the GW spectra are proportional to $v_{w}$ and the peak frequencies shift as $1 / v_{w}$. Therefore, one change in the wall velocity has, approximately, an order one effect on the spectrum and peak frequencies.

\section{Results and discussions}

The strength of the electroweak phase transition is quantified as

$$
\xi_{c}=\frac{v_{c}}{T_{c}},
$$

with $v_{c}=\sqrt{\left\langle h_{1}\right\rangle^{2}+\left\langle h_{2}\right\rangle^{2}}$ at critical temperature $T_{c}$. The global minimum of potential has $\langle A\rangle=0$ because of the $C P$-conserving case. In order to avoid washing out the baryon number generated during the phase transition, a SFOEWPT is required and the conventional condition is $\xi_{c} \geq 1$.

After imposing the constraints of "pre-LHC", the LHC Higgs data, the relic density, XENON1T, and Fermi-LAT, we scan over the parameter space in the previously selected scenario. We find some surviving samples which can achieve a SFOEWPT, and these samples are projected in Figs. 4 and 5. For all of the surviving samples, at $T_{c}$ the two degenerate minima of potential are at $\left(\left\langle h_{1}\right\rangle,\left\langle h_{2}\right\rangle, 0\right)$ and $(0$, $0,0)$, respectively. In the process of EWPT, $\left\langle S_{1}\right\rangle$ always has no VEV.

From Fig. 4, we find that $\left\langle h_{1}\right\rangle$ and $\left\langle h_{2}\right\rangle$ can vary in ranges from $20 \mathrm{GeV}$ to $150 \mathrm{GeV}$ and from $125 \mathrm{GeV}$ to 

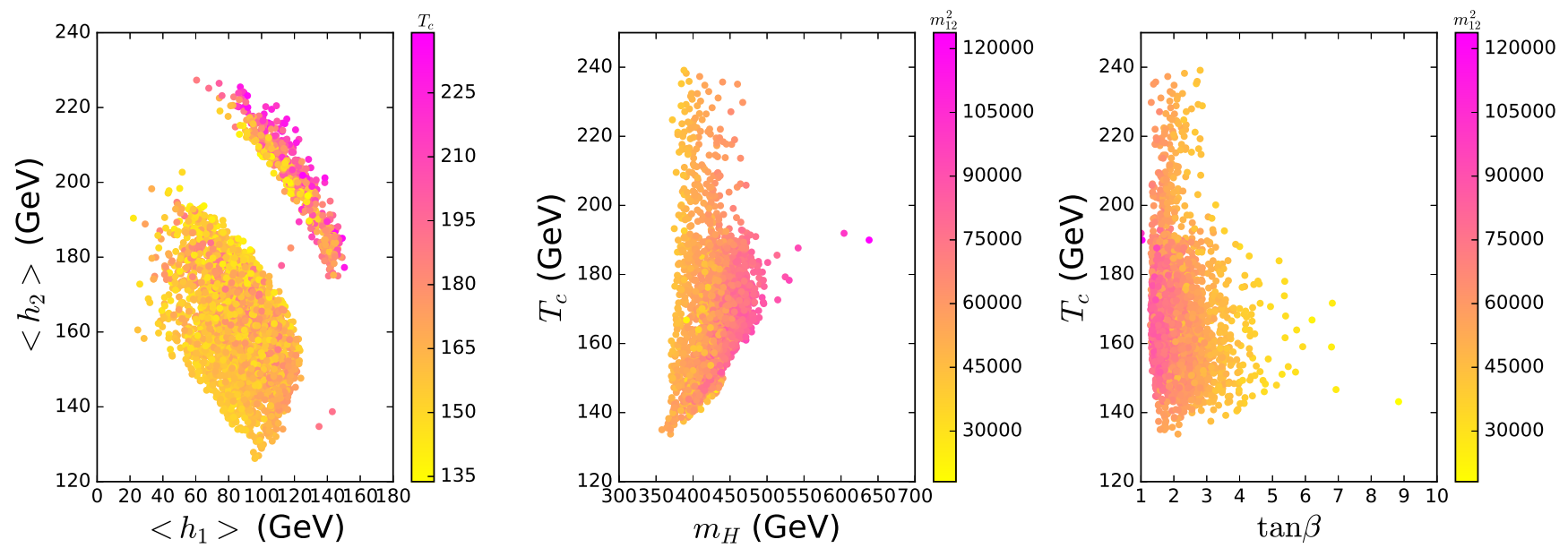

FIG. 4. The surviving samples projected on the planes of $\left\langle h_{1}\right\rangle$ versus $\left\langle h_{2}\right\rangle, T_{c}$ versus $m_{H}$, and $T_{c}$ versus $\tan \beta$. All of the samples achieve a SFOEWPT.
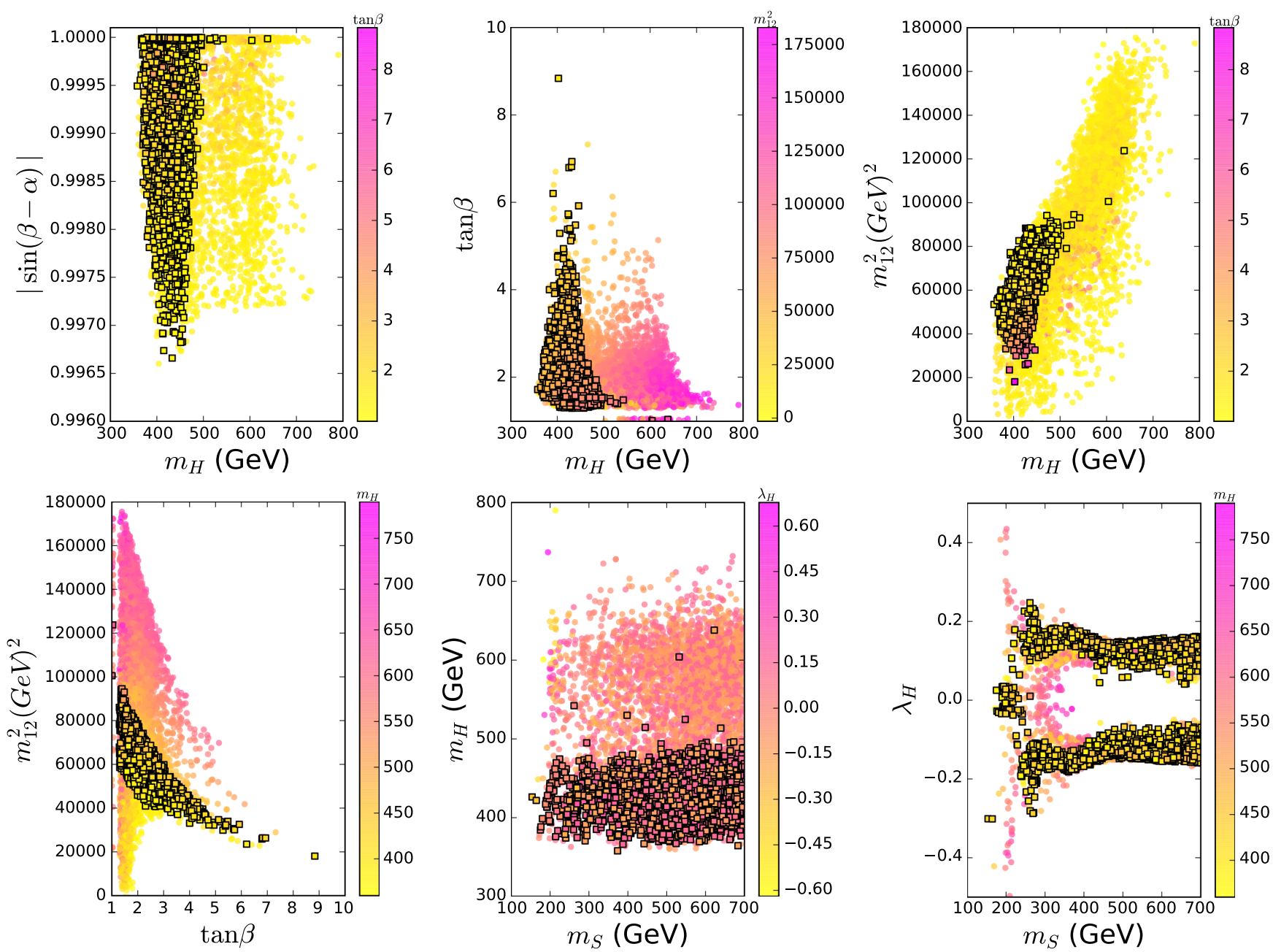

FIG. 5. The surviving samples projected on the planes of $\mid \sin (\beta-\alpha), \tan \beta, m_{12}^{2}$ versus $m_{H}, m_{12}^{2}$ versus $\tan \beta, m_{S}$ versus $m_{H}$, and $\lambda_{H}$. All of the samples are allowed by the constraints of "pre-LHC", the LHC Higgs data, and the DM observables. The squares achieve a SFOEWPT, and bullets fail. 
$230 \mathrm{GeV}$ with $T_{c}$ varying from $134 \mathrm{GeV}$ to $240 \mathrm{GeV} . T_{c}$ tends to increase with $m_{H}$, and has a relative small value for a large $\tan \beta$. It should also be noted that the relic abundance of the DM is achieved by the thermal freezeout in the early universe when the temperature was about $T \sim m_{S} / 25$. In the model, $T_{c}$ is much larger than $m_{S} / 25$ for $50 \mathrm{GeV}<m_{S}<700 \mathrm{GeV}$. Therefore, the EWPT hardly affects the thermal freeze-out process of DM.

From Fig. 5, we find that a SFOEWPT favors a small $m_{H}$, namely a large mass splitting between $m_{H}$ and $m_{A}$, which is consistent with Refs. [17,19]. Most of the samples lie in the region of $m_{H}<500 \mathrm{GeV}$, and there are several samples with $m_{H}>500 \mathrm{GeV}$ when $|\sin (\beta-\alpha)|$ is very close to 1.0. Also, a SFOEWPT favors $m_{12}^{2}$ to increase with $m_{H}$ and decrease with an increase of $\tan \beta$. There is a relatively strong correlation between $m_{12}^{2}$ and $\tan \beta$, and $m_{12}^{2}$ is imposed upper and lower bounds for a given $\tan \beta$. With an increase of $\tan \beta, m_{12}^{2}$ is stringently restricted by the theoretical constraints, leading that it is difficult to achieve a SFOEWPT. Thus, most of the samples lie in the region of the small $\tan \beta$. Also, similar results can be expected for type I 2HDM, especially for a large $m_{H}$. The requirement of a SFOEWPT is not sensitive to $m_{S}$, and disfavors $\left|\lambda_{H}\right|>0.3$.

In this model, due to the constraints of the DM relic density, generally the couplings $\kappa_{1}$ and $\kappa_{2}$ of the DM and $h_{1,2}$ are not large enough to give remarkable effects on the SFOEWPT compared to that of $2 \mathrm{HDM}$ without the singlet DM. Certainly, the $\lambda_{i}$ for the SFOEWPT may be slightly changed because of the presence of $\kappa_{1}$ and $\kappa_{2}$. Via the $h_{1,2^{-}}$ dependent non-SM Higgs squared masses, the thermal correction $V_{T}$ and the resummed daisy correction $V_{\text {ring }}$ give the cubic terms of $h_{1,2}$ proportional to T, which play key roles in generating a potential barrier and achieving the SFOEWPT. Schematically, the $h_{1,2}$-dependent squared masses of $H, A$, and $H^{ \pm}$can be given as [17,114]

$$
m_{\phi}^{2} \approx y_{\phi} \frac{m_{12}^{2}}{c_{\beta} s_{\beta}}+f_{\phi}\left(\lambda_{i}, h_{1}^{2}, h_{2}^{2}\right),
$$

with $y_{\phi}=1$ for $A, H^{ \pm}$and $y_{\phi}=\sin (\beta-\alpha)$ for $H$. When the term of $m_{12}^{2}$ dominates over $f_{\phi}\left(\lambda_{i}, h_{1}^{2}, h_{2}^{2}\right)$, the cubic terms in finite potential are suppressed. As a result, the mass spectrum of $H, A$, and $H^{ \pm}$tends to be degenerate, and the phase transition strength tends to be reduced. Conversely, when $f_{\phi}\left(\lambda_{i}, h_{1}^{2}, h_{2}^{2}\right)$ dominates over the term of $m_{12}^{2}$, the cubic terms are not suppressed. Therefore, the phase transition strength tends to be increased and the mass spectrum tends to be split.

There have been many studies of EWPT in the 2HDM [15-19]. Compared with previous studies, we comprehensively examine the current constraints on the parameter space using the latest heavy Higgs boson searches at LHC. Since the SFOEWPT is sensitive to the mass spectrum of

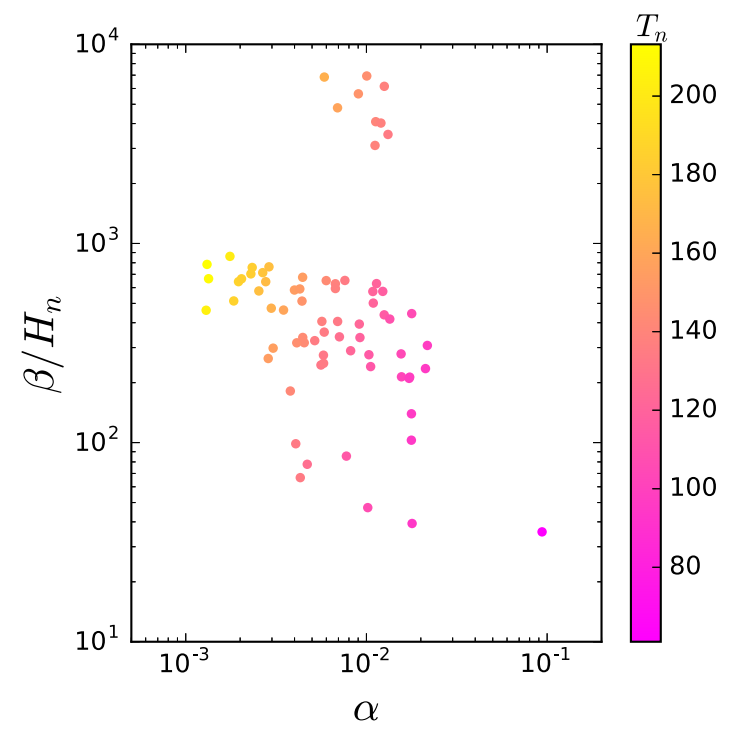

FIG. 6. The parameters $\alpha$ and $\beta / H_{n}$ characterizing the dynamics of the SFOEWPT.

the heavy Higgs bosons, the LHC searches for Higgs bosons can be used to reduce the parameter space achieving the SFOEWPT. For example, in this paper we find that the SFOEWPT is not achievable in the region of $m_{H}<$ $360 \mathrm{GeV}$ because of the constraints from the heavy Higgs boson searches at LHC.

Now we examine two key parameters, $\alpha$ and $\beta / H_{n}$, which characterize the dynamics of the SFOEWPT and govern the strength of GW spectra. A larger $\alpha$ and a smaller $\beta / H_{n}$ can lead to stronger GW signals. In addition to the conditions of the successful bubble nucleations, we require that

$$
\xi_{n}=\frac{v_{n}}{T_{n}} \geq 1
$$

with $v_{n}=\sqrt{\left\langle h_{1}\right\rangle^{2}+\left\langle h_{2}\right\rangle^{2}}$ at the nucleation temperature $T_{n}$. In fact, this is a more precise condition of SFOEWPT

TABLE III. Input and output parameters for two benchmark points for fixed $m_{h}=125 \mathrm{GeV}, m_{A}=600 \mathrm{GeV}$, and $\lambda_{h}=0$.

\begin{tabular}{lcc}
\hline \hline & BP1 & BP2 \\
\hline $\sin (\beta-\alpha)$ & 0.9998 & 0.9991 \\
$\tan \beta$ & 1.95 & 1.87 \\
$m_{H}(\mathrm{GeV})$ & 369.55 & 387.97 \\
$m_{H^{ \pm}}(\mathrm{GeV})$ & 620.8 & 618.31 \\
$m_{12}^{2}(\mathrm{GeV})^{2}$ & 53049.1 & 53649.1 \\
$m_{S}(\mathrm{GeV})$ & 479.2 & 501.7 \\
$\lambda_{H}$ & 0.133 & -0.129 \\
$\lambda_{S}$ & 12.3 & 10.93 \\
$T_{c}(\mathrm{GeV})$ & 135.7 & 160.0 \\
$T_{n}(\mathrm{GeV})$ & 61.0 & 95.0 \\
$\beta / H_{n}$ & 35.6 & 102.8 \\
$\alpha$ & 0.094 & 0.018 \\
\hline \hline
\end{tabular}




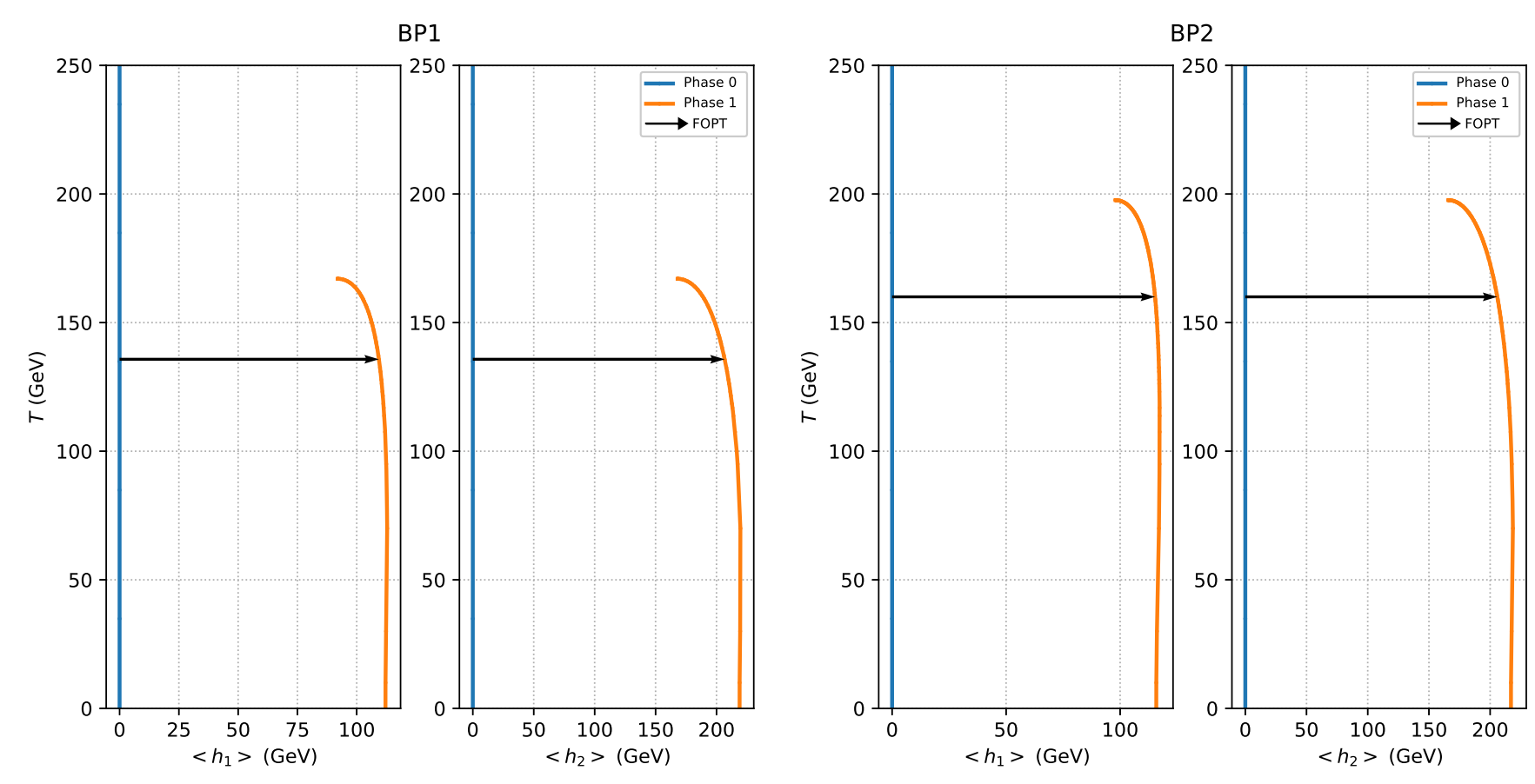

FIG. 7. Phase structures for BP1 (left) and BP2 (right). The lines show the field configurations at a particular minimum as a function of temperature. The arrows indicate that at that temperature $\left(T_{C}\right)$ the two phases linked by the arrows are degenerate, and can achieve the SFOEWPT.

than $\xi_{c} \geq 1$. Also note that there generically exists a difficulty for solving bounce solution in a very thin-walled bubble, including in the package CosmoTransitions [115]. Therefore, we will neglect the samples with a very thinwalled bubble. Considering the constraints discussed

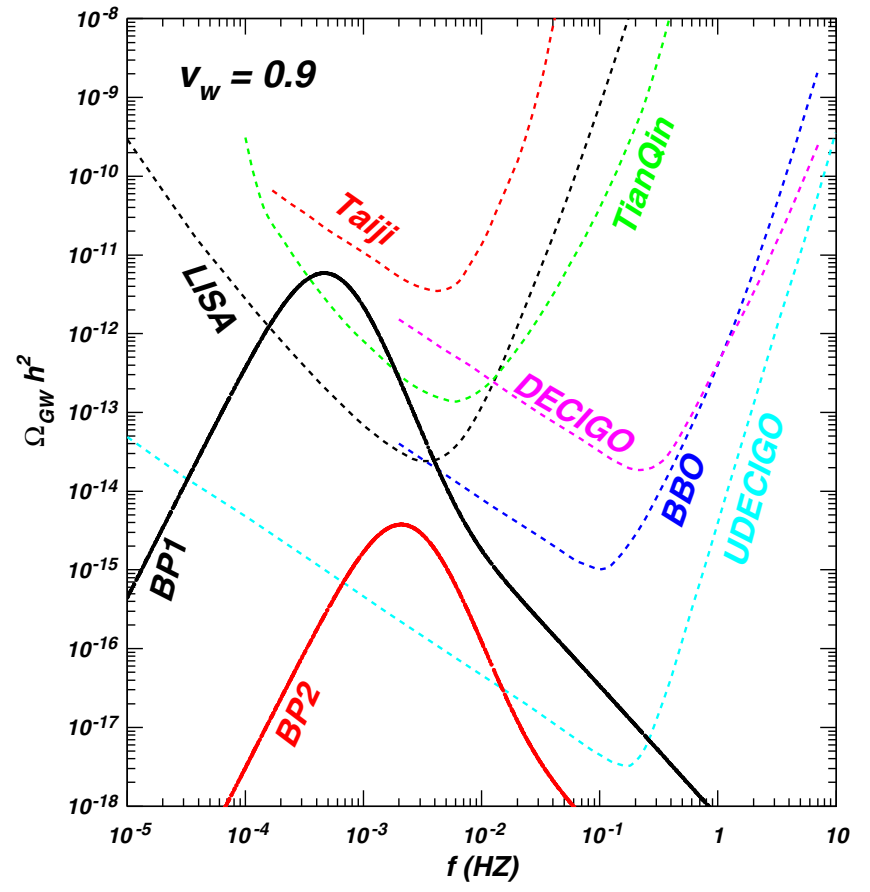

above, we find some surviving samples and the corresponding $\alpha$ and $\beta / H_{n}$ are shown in Fig. 6.

The $\beta / H_{n}$ may characterize the inverse time duration of the EWPT. A small $\beta / H_{n}$ means a long EWPT, and gives strong GW signals. For the GW coming from the sound

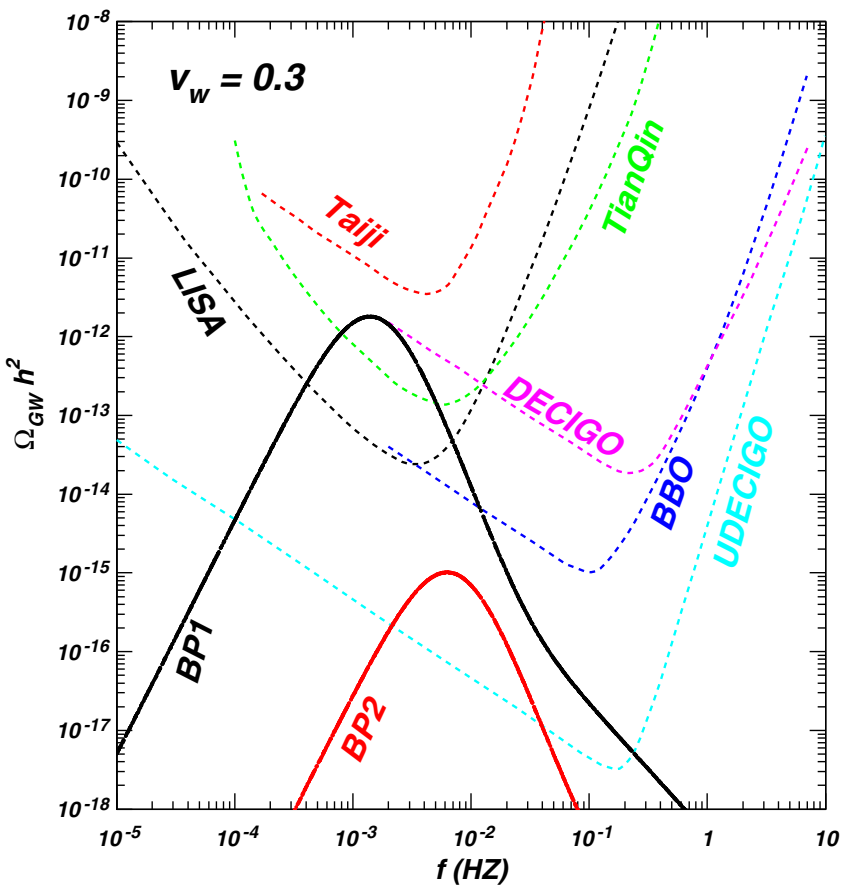

FIG. 8. Gravitational wave spectra for BP1 and BP2. 
waves in the plasma, the GW signal will continue being generated and the energy density of the GW is thus proportional to the duration of the EWPT if the mean square fluid velocity of the plasma is non-negligible [107]. In addition, a large $\beta / H_{n}$ can enhance the peak frequency of the GW spectra. The parameter $\alpha$ describes the amount of energy released during the EWPT, and therefore a large $\alpha$ leads to strong GW signals.

We pick out two benchmark points (BPs) and examine the corresponding GW spectra. Table III shows the input and output parameters of the BPs. Their phase histories are exhibited in Fig. 7 on field configurations versus the temperature plane. The field configuration $S_{1}$ is not shown as the minima at any temperatures located at $\left\langle S_{1}\right\rangle=0$. In Fig. 8, we show predicted GW spectra for our BPs along with expected sensitivities of various future interferometer experiments, and find that the amplitudes of the $\mathrm{GW}$ spectra reach the sensitivities of LISA, TianQin, BBO, DECIGO, and UDECIGO for BP1 (UDECIGO for BP2).

\section{CONCLUSION}

We examine the status of the 2HDMIID confronted with the recent LHC Higgs data, the DM observables, and SFOEWPT, and discuss the detectability of GW at the future GW detectors. We choose the heavy $C P$-even Higgs boson $H$ as the only portal between the DM and SM sectors, and focus on the case of the $125 \mathrm{GeV}$ Higgs with the SM-like coupling. We find that for $m_{A}=600 \mathrm{GeV}$, $m_{S}<130 \mathrm{GeV}$ and $m_{H}<360 \mathrm{GeV}$ are excluded by the joint constraints of the $125 \mathrm{GeV}$ Higgs signal data, the searches for additional Higgs via $H / A \rightarrow \tau^{+} \tau^{-}, A \rightarrow H Z$, $H \rightarrow W W, Z Z, \gamma \gamma, h h$ at the LHC, as well as the relic density XENON1T.

A SFOEWPT can be achieved in the many regions of $m_{H}<500 \mathrm{GeV}$ and $m_{A}=600 \mathrm{GeV}$, favors a small $\tan \beta$, and is not sensitive to the mass of DM. We find the benchmark points for which the predicted GW spectra can reach the sensitivities of LISA, TianQin, BBO, DECIGO, and UDECIGO.

\section{ACKNOWLEDGMENTS}

We thank L. Bian, Wei Chao and Huai-Ke Guo for helpful discussions. This work was supported by the National Natural Science Foundation of China under Grant No. 11975013, by the Natural Science Foundation of Shandong province (Grants No. ZR2017MA004 and ZR2017JL002), and by the ARC Centre of Excellence for Particle Physics at the Tera-scale under Grant No. CE110001004. This work is also supported by the Project of Shandong Province Higher Educational Science and Technology Program under Grant No. 2019KJJ007.
[1] T. D. Lee, Phys. Rev. D 8, 1226 (1973).

[2] H. E. Haber, G. L. Kane, and T. Sterling, Nucl. Phys. 161, 493 (1979).

[3] J. F. Donoghue and L. F. Li, Phys. Rev. D 19, 945 (1979).

[4] X.-G. He, T. Li, X.-Q. Li, J. Tandean, and H.-C. Tsai, Phys. Rev. D 79, 023521 (2009).

[5] X.-G. He and J. Tandean, Phys. Rev. D 88, 013020 (2013).

[6] Y. Cai and T. Li, Phys. Rev. D 88, 115004 (2013).

[7] L. Wang and X.-F. Han, Phys. Lett. B 739, 416 (2014).

[8] A. Drozd, B. Grzadkowski, J. F. Gunion, and Y. Jiang, J. High Energy Phys. 11 (2014) 105.

[9] X.-G. He and J. Tandean, J. High Energy Phys. 12 (2016) 074.

[10] T. Alanne, K. Kainulainen, K. Tuominen, and V. Vaskonen, J. Cosmol. Astropart. Phys. 08 (2016) 057.

[11] L. Wang, R. Shi, and X.-F. Han, Phys. Rev. D 96, 115025 (2017).

[12] N. Chen, Z. Kang, and J. Li, Phys. Rev. D 95, 015003 (2017).

[13] L. Wang, X.-F. Han, and B. Zhu, Phys. Rev. D 98, 035024 (2018).

[14] S. Baum and N. R. Shah, J. High Energy Phys. 12 (2018) 044.

[15] A. I. Bochkarev, S. V. Kuzmin, and M. E. Shaposhnikov, Phys. Lett. B 244, 275 (1990); J. M. Cline and
P.-A. Lemieux, Phys. Rev. D 55, 3873 (1997); G. C. Dorsch, S. J. Huber, and J. M. No, J. High Energy Phys. 10 (2013) 029; G. C. Dorsch, S. J. Huber, K. Mimasu, and J. M. No, Phys. Rev. Lett. 113, 211802 (2014); L. Wang, J. M. Yang, M. Zhang, and Y. Zhang, Phys. Lett. B 788, 519 (2019); N. Chen, T. Li, Z. Teng, and Y. Wu, J. High Energy Phys. 10 (2020) 081; R. Zhou and L. Bian, arXiv:2001.01237; R. Zhou, L. Bian, and H.-K Guo, Phys. Rev. D 101, 091903 (2020); X. Wang, F. Huang, and X. Zhang, Phys. Rev. D 101, 015015 (2020); J. Cosmol. Astropart. Phys. 05 (2020) 045.

[16] J. M. Cline, K. Kainulainen, and M. Trott, J. High Energy Phys. 11 (2011) 089.

[17] P. Basler, M. Krause, M. Muhlleitner, J. Wittbrodt, and A. Wlotzka, J. High Energy Phys. 02 (2017) 121.

[18] J. O. Andersen, T. Gorda, A. Helset, L. Niemi, and T. V. I. Tenkanen, Phys. Rev. Lett. 121, 191802 (2018).

[19] J. Bernon, L. Bian, and Y. Jiang, J. High Energy Phys. 05 (2018) 151.

[20] A. D. Sakharov, Pis'ma Zh. Eksp. Teor. Fiz. 5, 32 (1967) [JETP Lett. 5, 24 (1967)]; Usp. Fiz. Nauk 161, 61 (1991) [Sov. Phys. Usp. 34, 392 (1991)].

[21] M. Kamionkowski, A. Kosowsky, and M. S. Turner, Phys. Rev. D 49, 2837 (1994).

[22] H. Audley et al. (LISA Collaboration), arXiv:1702.00786. 
[23] X. Gong et al., J. Phys. Conf. Ser. 610, 012011 (2015).

[24] J. Luo et al. (TianQin Collaboration), Classical Quantum Gravity 33, 035010 (2016).

[25] K. Yagi and N. Seto, Phys. Rev. D 83, 044011 (2011).

[26] H. Kudoh, A. Taruya, T. Hiramatsu, and Y. Himemoto, Phys. Rev. D 73, 064006 (2006).

[27] R. A. Battye, G. D. Brawn, and A. Pilaftsis, J. High Energy Phys. 08 (2011) 020.

[28] Heavy Flavor Averaging Group, Eur. Phys. J. C 77, 895 (2017); M. Misiak and M. Steinhauser, Eur. Phys. J. C 77, 201 (2017).

[29] F. Kling, J. M. No, and S. Su, J. High Energy Phys. 09 (2016) 093.

[30] L. Wang, F. Zhang, and X.-F. Han, Phys. Rev. D 95, 115014 (2017).

[31] L. Wang, H.-X. Wang, and X.-F. Han, Chin. Phys. C 44, 073101 (2020).

[32] D. Eriksson, J. Rathsman, and O. Stål, Comput. Phys. Commun. 181, 189 (2010).

[33] M. Tanabashi et al. (Particle Data Group), Phys. Rev. D 98, 030001 (2018).

[34] F. Mahmoudi, Comput. Phys. Commun. 180, 1579 (2009).

[35] C. Q. Geng and J. N. Ng, Phys. Rev. D 38, 2857 (1988); 41, 1715(E) (1990).

[36] H. E. Haber and H. E. Logan, Phys. Rev. D 62, 015011 (2000).

[37] G. Degrassi and P. Slavich, Phys. Rev. D 81, 075001 (2010).

[38] J. Bernon, B. Dumont, and S. Kraml, Phys. Rev. D 90, 071301 (2014); S. Kraml, T. Q. Loc, D. T Nhung, and L. D. Ninh, SciPost Phys. 7, 052 (2019).

[39] P. Bechtle, O. Brein, S. Heinemeyer, G. Weiglein, and K. E. Williams, Comput. Phys. Commun. 181, 138 (2010).

[40] P. Bechtle, O. Brein, S. Heinemeyer, O. Stål, T. Stefaniak, G. Weiglein, and K. E. Williams, Eur. Phys. J. C 74, 2693 (2014).

[41] R. V. Harlander, S. Liebler, and H. Mantler, Comput. Phys. Commun. 184, 1605 (2013).

[42] S. Heinemeyer et al. (LHC Higgs Cross Section Working Group Collaboration), arXiv:1307.1347.

[43] S. Moretti, Proc. Sci., CHARGED2016 (2016) 014.

[44] G. Aad et al. (ATLAS Collaboration), J. High Energy Phys. 11 (2014) 056.

[45] CMS Collaboration, Search for additional neutral Higgs bosons decaying to a pair of tau leptons in $p p$ collisions at $\sqrt{s}=7$ and $8 \mathrm{TeV}$, CMS-PAS-HIG-14-029.

[46] CMS Collaboration, Search for a neutral MSSM Higgs Boson decaying into $\tau \tau H / A$ with $12.9 \mathrm{fb}^{-1}$ of data at $\sqrt{s}=13 \mathrm{TeV}$, CMS-PAS-HIG-16-037.

[47] ATLAS Collaboration, J. High Energy Phys. 01 (2018) 055.

[48] CMS Collaboration, Phys. Lett. B 758, 296 (2016).

[49] ATLAS Collaboration, Phys. Rev. Lett. 125, 051801 (2020).

[50] CMS Collaboration, Search for a light pseudoscalar Higgs boson produced in association with bottom quarks in $\mathrm{pp}$ collisions at $\sqrt{s}=8 \mathrm{TeV}$, CMS-HIG-15-009.

[51] ATLAS Collaboration, Search for scalar diphoton resonances with $15.4 \mathrm{fb}^{-1}$ of data collected at $\sqrt{s}=13 \mathrm{TeV}$ in
2015 and 2016 with the ATLAS detector, ATLAS-CONF2016-059.

[52] CMS Collaboration, Search for resonant production of high mass photon pairs using $12.9 \mathrm{fb}^{-1}$ of proton-proton collisions at $\sqrt{s}=13 \mathrm{TeV}$ and combined interpretation of searches at 8 and $13 \mathrm{TeV}$, CMS-PAS-EXO-16-027.

[53] CMS Collaboration, Search for new resonances in the diphoton final state in the mass range between 70 and $110 \mathrm{GeV}$ in pp collisions at $\sqrt{s}=8$ and $13 \mathrm{TeV}$, CMSPAS-HIG-17-013.

[54] G. Aad et al. (ATLAS Collaboration), J. High Energy Phys. 01 (2016) 032.

[55] ATLAS Collaboration, Search for a high-mass Higgs boson decaying to a pair of $\mathrm{W}$ bosons in pp collisions at $\sqrt{s}=13 \mathrm{TeV}$ with the ATLAS detector, ATLASCONF-2016-074.

[56] ATLAS Collaboration, Search for diboson resonance production in the $\ell \nu q q$ final state using p p collisions at $\sqrt{s}=13 \mathrm{TeV}$ with the ATLAS detector at the LHC, ATLAS-CONF-2016-062.

[57] ATLAS Collaboration, J. High Energy Phys. 03 (2018) 042.

[58] ATLAS Collaboration, Eur. Phys. J. C 78, 24 (2018).

[59] CMS Collaboration, J. High Energy Phys. 03 (2020) 034.

[60] G. Aad et al. (ATLAS Collaboration), Eur. Phys. J. C 76, 45 (2016).

[61] ATLAS Collaboration, Search for new phenomena in the $Z(\rightarrow \ell \ell)+E_{\mathrm{T}}^{\text {miss }}$ final state at $\sqrt{s}=13 \mathrm{TeV}$ with thee ATLAS detector, ATLAS-CONF-2016-056.

[62] ATLAS Collaboration, Searches for heavy ZZ and $\mathrm{ZW}$ resonances in the $\ell \ell q q$ and vvqq final states in $\mathrm{pp}$ collisions at $\sqrt{s}=13 \mathrm{TeV}$ with the ATLAS detector, ATLAS-CONF-2016-082.

[63] ATLAS Collaboration, Study of the Higgs boson properties and search for high-mass scalar resonances in the $H \rightarrow Z Z^{*} \rightarrow 4 \ell$ decay channel at $\sqrt{s}=13 \mathrm{TeV}$ with the ATLAS detector, ATLAS-CONF-2016-079.

[64] ATLAS Collaboration, Eur. Phys. J. C 78, 293 (2018).

[65] ATLAS Collaboration, J. High Energy Phys. 03 (2018) 009.

[66] V. Khachatryan et al. (CMS Collaboration), Phys. Rev. D 94, 052012 (2016).

[67] V. Khachatryan et al. (CMS Collaboration), Phys. Lett. B 749, 560 (2015).

[68] V. Khachatryan et al. (CMS Collaboration), Phys. Lett. B 755, 217 (2016).

[69] ATLAS Collaboration, Search for pair production of Higgs bosons in the $b \bar{b} b \bar{b}$ final state using proton-proton collisions at $\sqrt{s}=13 \mathrm{TeV}$ with the ATLAS detector, ATLASCONF-2016-049.

[70] CMS Collaboration, Phys. Lett. B 781, 244 (2018).

[71] CMS Collaboration, Phys. Lett. B 778, 101 (2018).

[72] CMS Collaboration, Phys. Rev. Lett. 122, 121803 (2019).

[73] CMS Collaboration, Phys. Rev. D 102, 032003 (2020).

[74] ATLAS Collaboration, J. High Energy Phys. 11 (2020) 163.

[75] V. Khachatryan et al. (CMS Collaboration), Phys. Lett. B 748, 221 (2015).

[76] G. Aad et al. (ATLAS Collaboration), Phys. Lett. B 744, 163 (2015). 
[77] ATLAS Collaboration, J. High Energy Phys. 03 (2018) 174.

[78] CMS Collaboration, Eur. Phys. J. C 79, 564 (2019).

[79] CMS Collaboration, J. High Energy Phys. 03 (2020) 065.

[80] ATLAS Collaboration, Phys. Rev. D 92, 052002 (2015).

[81] CMS Collaboration, J. High Energy Phys. 10 (2017) 076.

[82] CMS Collaboration, Phys. Lett. B 785, 462 (2018).

[83] CMS Collaboration, Phys. Lett. B 800, 135087 (2020).

[84] CMS Collaboration, J. High Energy Phys. 08 (2020) 139.

[85] V. Khachatryan et al. (CMS Collaboration), Phys. Lett. B 759, 369 (2016).

[86] ATLAS Collaboration, Phys. Lett. B 783, 392 (2018).

[87] CMS Collaboration, J. High Energy Phys. 03 (2020) 055.

[88] F. Kling, J. M. No, and S. Su, J. High Energy Phys. 09 (2016) 093.

[89] A. Alloul, N. D. Christensen, C. Degrande, C. Duhr, and B. Fuks, Comput. Phys. Commun. 185, 2250 (2014).

[90] G. Belanger, F. Boudjema, A. Pukhov, and A. Semenov, Comput. Phys. Commun. 185, 960 (2014).

[91] G. Jungman, M. Kamionkowski, and K. Griest, Phys. Rep. 267, 195 (1996); M. A. Shifman, A. I. Vainshtein, and V. I. Zakharov, Phys. Lett. B 78, 443 (1978).

[92] Planck Collaboration, Astron. Astrophys. 594, A24 (2016).

[93] E. Aprile et al. (XENON Collaboration), Phys. Rev. Lett. 121, 111302 (2018).

[94] Fermi-LAT Collaboration, Phys. Rev. Lett. 115, 231301 (2015).

[95] S. R. Coleman and E. J. Weinberg, Phys. Rev. D 7, 1888 (1973).

[96] L. Dolan and R. Jackiw, Phys. Rev. D 9, 3320 (1974).

[97] M. E. Carrington, Phys. Rev. D 45, 2933 (1992).
[98] P. B. Arnold and O. Espinosa, Phys. Rev. D 47, 3546 (1993); 50, 6662 (1994).

[99] I. Affleck, Phys. Rev. Lett. 46, 388 (1981).

[100] A. D. Linde, Nucl. Phys. B216, 421 (1983); B223, 544 (1983)].

[101] A. D. Linde, Phys. Lett. B 100, 37 (1981).

[102] C. L. Wainwright, Comput. Phys. Commun. 183, 2006 (2012).

[103] P. Athron, C. Balázs, A. Fowlie, and Y. Zhang, Eur. Phys. J. C 80, 567 (2020).

[104] D. Bodeker and G. D. Moore, J. Cosmol. Astropart. Phys. 05 (2009) 009.

[105] D. Bodeker and G. D. Moore, J. Cosmol. Astropart. Phys. 05 (2017) 025.

[106] D. Bodeker and G. D. Moore, J. Cosmol. Astropart. Phys. 05 (2017) 025.

[107] M. Hindmarsh, S. J. Huber, K. Rummukainen, and D. J. Weir, Phys. Rev. D 92, 123009 (2015).

[108] J. Kozaczuk, J. High Energy Phys. 10 (2015) 135.

[109] J. M. No, Phys. Rev. D 84, 124025 (2011).

[110] J. R. Espinosa, T. Konstandin, J. M. No, and G. Servant, J. Cosmol. Astropart. Phys. 06 (2010) 028.

[111] A. Kosowsky, A. Mack, and T. Kahniashvili, Phys. Rev. D 66, 024030 (2002).

[112] C. Caprini, R. Durrer, and G. Servant, J. Cosmol. Astropart. Phys. 12 (2009) 024.

[113] P. Binetruy, A. Bohe, C. Caprini, and J.-F. Dufaux, J. Cosmol. Astropart. Phys. 06 (2012) 027.

[114] M Krause, M. Muhlleitner, R. Santos, and H. Ziesche, Phys. Rev. D 95, 075019 (2017).

[115] P. Athron, C. Balázs, M. Bardsley, A. Fowlie, D. Harries, and G. White, Comput. Phys. Commun. 244, 448 (2019). 